\title{
GLOBAL AND NONGLOBAL PARAMETERS OF HORIZONTAL-BRANCH MORPHOLOGY OF GLOBULAR CLUSTERS*
}

\author{
A. P. Milone ${ }^{1,2,3}$, A. F. Marino ${ }^{1}$, A. Dotter ${ }^{1}$, J. E. Norris ${ }^{1}$, H. Jerjen ${ }^{1}$, G. Piotto ${ }^{4,5}$, S. CAssisi ${ }^{6}$, L. R. Bedin ${ }^{5}$, \\ A. Recio Blanco ${ }^{7}$, A. Sarajedini ${ }^{8}$, M. Asplund ${ }^{1}$, M. Monelli ${ }^{2,3}$, And A. Aparicio ${ }^{2,3}$ \\ ${ }^{1}$ Research School of Astronomy and Astrophysics, The Australian National University, Cotter Road, Weston, ACT, 2611, Australia; milone@mso.anu.edu.au, \\ amarino@mso.anu.edu.au,jen@mso.anu.edu.au, jerjen@mso.anu.edu.au, martin@mso.anu.edu.au \\ ${ }^{2}$ Instituto de Astrofisica de Canarias, E-38200 La Laguna, Tenerife, Canary Islands, Spain; monelli@iac.es, aparicio@iac.es \\ ${ }^{3}$ Department of Astrophysics, University of La Laguna, E-38200 La Laguna, Tenerife, Canary Islands, Spain \\ ${ }^{4}$ Dipartimento di Fisica e Astronomia, “Galileo Galilei”" Università di Padova, Vicolo dell'Osservatorio 3, Padova I-35122, Italy; giampaolo.piotto@ unipd.it \\ ${ }^{5}$ INAF-Osservatorio Astronomico di Padova, Vicolo dell'Osservatorio 5, I-35122 Padua, Italy; luigi.bedin@oapd.inaf.it \\ ${ }^{6}$ INAF-Osservatorio Astronomico di Collurania, via Mentore Maggini, I-64100 Teramo, Italy; cassisi@ oa-teramo.inaf.it \\ ${ }^{7}$ Observatoire de la Cote d'Azur, UMR Cassiopee, B. P. 4229, F-06304 Nice Cedex 04, France; alejandra.recio-blanco@ oca.eu \\ ${ }^{8}$ Department of Astronomy, University of Florida, 211 Bryant Space Science Center, Gainesville, FL 32611, USA; ata@ astro.ufl.edu \\ Received 2013 May 15; accepted 2013 December 12; published 2014 March 21
}

\begin{abstract}
The horizontal-branch (HB) morphology of globular clusters (GCs) is mainly determined by metallicity. However, the fact that GCs with almost the same metallicity exhibit different HB morphologies demonstrates that at least one more parameter is needed to explain the HB morphology. It has been suggested that one of these should be a global parameter that varies from GC to GC and the other a nonglobal parameter that varies within the GC. In this study we provide empirical evidence corroborating this idea. We used the photometric catalogs obtained with the Advanced Camera for Surveys of the Hubble Space Telescope and analyze the color-magnitude diagrams of 74 GCs. The HB morphology of our sample of GCs has been investigated on the basis of the two new parameters $L 1$ and $L 2$ that measure the distance between the red giant branch and the coolest part of the HB and the color extension of the $\mathrm{HB}$, respectively. We find that $L 1$ correlates with both metallicity and age, whereas $L 2$ most strongly correlates with the mass of the hosting GC. The range of helium abundance among the stars in a GC, characterized by $\Delta Y$ and associated with the presence of multiple stellar populations, has been estimated in a few GCs to date. In these GCs we find a close relationship among $\Delta Y$, GC mass, and $L 2$. We conclude that age and metallicity are the main global parameters, while the range of helium abundance within a GC is the main nonglobal parameter defining the HB morphology of Galactic GCs.
\end{abstract}

Key words: globular clusters: general - stars: abundances - stars: Population II

Online-only material: color figures

\section{INTRODUCTION}

Since the early 1950 s, metallicity has been considered the main parameter that determines the horizontal-branch (HB) morphology in globular clusters (GCs; e.g., Arp et al. 1952). Within a few years, evidence that some GCs with similar metallicity exhibit different HB morphologies suggested that at least a second parameter (2ndP) is required to properly characterize the HB morphology of GCs (e.g., Sandage \& Wallerstein 1960; van den Bergh 1965). Since then, the so-called 2 ndP problem has been widely investigated by many authors.

Several candidates have been suggested as possible $2 \mathrm{ndPs}$, including mass loss (e.g., Peterson 1982; Catelan 2000), stellar rotation (e.g., Mengel \& Gross 1976; Fusi-Pecci \& Renzini 1978), planetary systems (e.g., Soker 1998; Siess \& Livio 1999), magnetic fields (e.g., Rood \& Seitzer 1981), and GC ellipticity (Norris 1983), but a comprehensive picture is still lacking. Age (e.g., Searle \& Zinn 1978; Catelan \& de Freitas Pacheco 1993; Lee et al. 1994), GC central density (e.g., Fusi Pecci et al. 1993), GC mass (e.g., Recio-Blanco et al. 2006), and helium abundance (e.g., Sandage \& Wildey 1967; van den Bergh 1967) are among the best candidates. We refer the reader to the papers by Freeman \& Norris (1981), Catelan (2009), Dotter et al.

\footnotetext{
* Based on observations with the NASA/ESA Hubble Space Telescope, obtained at the Space Telescope Science Institute, which is operated by AURA, Inc., under NASA contract NAS 5-26555.
}

(2010), and Gratton et al. (2010) and references therein for reviews on HB stars and the 2ndP phenomenon in GCs.

The study of GCs has changed dramatically in recent years because of the overwhelming evidence for the existence of multiple stellar populations in GCs. In this way of thinking, a GC is made up of a first generation of stars, formed from the GC's primordial gas cloud, and at least one later generation, formed from a dilution of the primordial gas and the chemical yields of the high- and intermediate-mass stars of the first generation.

The possibility of GC self-enrichment, especially as it relates to enhanced helium, as the cause for the variation of the HB morphology has been investigated by several authors, as multiple stellar populations with different helium abundance can indeed explain features such as tails and multimodalities in the HBs of GCs (e.g., Ferraro et al. 1998; D'Antona et al. 2002, 2005; Piotto et al. 2007; Caloi \& D'Antona 2008; Gratton et al. 2010). The idea of a connection between multiple stellar populations and HB morphology arose in the early 1980s, when pioneering papers showed that the cyanogen distribution is closely connected to the shape of the HB (e.g., Norris 1981; Norris et al. 1981; Smith \& Norris 1993); this result has been confirmed by recent studies of HB stars.

In this context the GC M 4 is exemplary. High-resolution spectroscopy of red giant branch (RGB) stars reveals that this GC hosts two stellar populations with different $\mathrm{Na}$ and $\mathrm{O}$ abundances, while photometry reveals two RGBs in the $U$ versus $U-B$ color-magnitude diagram (CMD). The HB of M 4 is 
bimodal and well-populated on both sides of the RR Lyrae gap (Marino et al. 2008). The bimodality in $\mathrm{Na}$ and $\mathrm{O}$ is also present among the HB stars. Blue-HB stars belong to the second population and are O-poor and Na-rich, while red HB stars are first population (Marino et al. 2008, 2011b). Similar analyses of $\mathrm{Na}$ and $\mathrm{O}$ in HB stars in other GCs show that first-generation HB stars preferentially populate the reddest HB segment, while second-generation HB stars tend toward bluer colors (Villanova et al. 2009; Gratton et al. 2011, 2012, 2013; Lovisi et al. 2012; Marino et al. 2013). More recently (Marino et al. 2014) inferred from direct spectroscopic measurements that Na-rich HB stars of NGC 2808 are also strongly helium enhanced.

Several factors influence HB morphology, and it is difficult to disentangle the different effects. An important point in the study of the $2 \mathrm{ndP}$ is that the metric used to characterize HB morphology is not objective; the chosen way of representing the $\mathrm{HB}$ stars in a $\mathrm{GC}$ as a number has a nontrivial influence on the results of the investigation. Most studies to date adopt a single HB morphology metric. Different studies, using different metrics, can easily reach conflicting conclusions about the identity of the 2 ndP.

Consider the following two examples. Recio-Blanco et al. (2006) defined their HB morphology metric as the maximum effective temperature along the HB and found that more massive GCs tend to have hotter HBs. Dotter et al. (2010) measured the median color difference between the $\mathrm{HB}$ and the RGB at the level of the HB $(\Delta(V-I))$ from Hubble Space Telescope (HST) Advanced Camera for Surveys (ACS) photometry of $60 \mathrm{GCs}$ and demonstrated that after the metallicity dependence is accurately removed, $\Delta(V-I)$ correlates most strongly with GC age.

Dotter et al. (2010, see their Figure 2) compared $\Delta(V-I)$ with the widely used HB Type index ${ }^{9}$ and the maximum effective temperature along the HB as defined by Recio-Blanco et al. (2006). The comparison shows that $\Delta(V-I)$ and HB Type are closely correlated but that $\Delta(V-I)$ and Recio-Blanco et al.'s maximum HB temperatures are not: they have very different sensitivities. It is not surprising that a metric sensitive to the extremes of the distribution correlates with a different (second) parameter than a metric sensitive to the center of the distribution. The problem is that $2 \mathrm{ndP}$ studies typically select only one HB morphology metric and the conclusions are influenced by that choice.

We suggest that a more effective way to proceed is to consider more than one HB morphology metric simultaneously. For maximum effect, these metrics should share a simple, common definition but not be closely correlated with each other. The motivation for this approach is not only based on the practicalities outlined above. Freeman \& Norris (1981) argued that two parameters, one global and one local, may be needed to fully describe the observed variations in HB morphology. The local parameter is one that varies within a single GC; the global parameter is one that varies among the GC population.

The aim of this paper is an empirical investigation of the parameters governing the $\mathrm{HB}$ morphology of GCs, in the context of the classical 2ndP phenomenon. To do this we use the homogeneous high-accuracy photometry from GO 10775, the ACS Survey of Galactic GCs (PI: A. Sarajedini; Sarajedini et al. 2007), and from GO 11586 (PI: A. Dotter; Dotter et al. 2011), and additional photometry from HST to reinvestigate the HB

\footnotetext{
9 Defined as $(B-R) /(B+V+R)$, where $B, R$, and $V$ are the numbers of blue HB, red HB, and variables stars. It measures the relative contributions of stars bluer or redder than the RR Lyrae instability strip and is known variously as HB Type and HB Ratio (HBR)
}

morphology in GCs in light of the new findings on multiple stellar populations in GCs and of the global versus nonglobal parameter idea by Freeman \& Norris (1981). The paper is organized as follows. Section 2 describes the observational data. Section 3 introduces the quantities adopted to describe the HB morphology and defines the new HB morphology parameters $L 1$ and $L 2$. Section 4 assembles a variety of GC parameters from the literature. Section 5 compares these parameters with $L 1$ and L2. Section 6 discusses our findings in the context of similar studies in the literature. Finally, we summarize our findings in Section 7.

\section{THE DATA SAMPLE}

We used the photometric catalogs obtained from GO 10775 and GO 11586 that include homogeneous photometric and astrometric measurements for 65 and $6 \mathrm{GCs}$, respectively. For each of them, the data set consists of one short and four or five long exposures in the F606W and F814W bandpasses. We excluded from GO 10775 three GCs: Pal 1 and E 3 for the lack of identifiable HB stars and Pal 2 for the extreme differential reddening. The details concerning the data, the data reduction, and the calibration are given in Anderson et al. (2008) and Dotter et al. (2011).

In their study of the HB in GCs, Dotter et al. (2010) emphasized the importance of properly accounting for the outer halo, where the $2 \mathrm{ndP}$ is more evident. To increase the number of outer-halo GCs, we have extended the GO 10775 and GO 11586 to six more GCs: AM-1, Eridanus, NGC 2419, Pal 3, Pal 4, and Pal 14. For both Pal 4 and Pal 14, we used $2 \times 60$ s F606W and $2 \times 80 \mathrm{~s}$ F814W ACS/WFC images from GO 10622 (PI: Dolphin), while for NGC 2419 we used the F606W and F814W magnitudes published by di Criscienzo et al. (2011). These data have been reduced as already described in Anderson et al. (2008), Dotter et al. (2011), and di Criscienzo et al. (2011). For Pal 3 and Eridanus we used ground-based $V, I$ photometry from Stetson et al. (1999) and for AM-1 Wide Field Planetary Camera 2 (WFPC2) photometry from Dotter et al. (2008) in F555W and F814W. Photometry for these three GCs has been transformed into F606W and F814W ACS/WFC bands by using the relationships given in Sirianni et al. (2005).

Photometry has been corrected for spatial photometric zeropoint variation both due to differential reddening and small inaccuracies in the point-spread function model (Anderson et al. 2008). For most GCs we used the corrected magnitudes and colors published by Milone et al. (2012b; 59 GCs), Piotto et al. (2012; NGC 6715), di Criscienzo et al. (2011; NGC 2419), and Bellini et al. (2010; $\omega$ Centauri). For the remaining GCs we corrected the photometry following the procedure described in Milone et al. (2012b).

\section{TWO NEW PARAMETERS TO DESCRIBE THE HB MORPHOLOGY: $L 1$ AND $L 2$}

To investigate the HB morphology, we defined two quantities: $L 1$, the color difference between the RGB and the coolest border the $\mathrm{HB}$, and $L 2$, the color extension of the HB. ${ }^{10}$

\footnotetext{
10 We emphasize that we have not introduced $L 1$ and $L 2$ because we believe that they are more (or less) efficient than any other diagnostic previously used in the study of the HB morphology. Nevertheless, results of the empirical analysis presented in this paper will show that $L 1$ and $L 2$ are useful tools to shed light on the 2ndP phenomenon. Any search for the best diagnostic of the HB morphology is obviously outside the scope of our paper and is possibly naive; one would need to understand a priori which parameters determine $\mathrm{HB}$ morphology and then compare the sensitivity of $L 1, L 2$, and the other diagnostics of the HB morphology used in the literature with these parameters.
} 
The procedure to determine $L 1$ and $L 2$ is illustrated in Figure 1 for the case of NGC 5904 (M 5). We selected by eye a sample of HB stars that we plotted as blue circles in the lower panel and a sample of RGB stars that we represented with red points. The RGB sample includes all the RGB stars with luminosity differing by less than $\pm 0.1 \mathrm{~F} 606 \mathrm{~W}$ mag from the mean level of the $\mathrm{HB}\left(\mathrm{F} 606 \mathrm{~W}_{\mathrm{HB}}\right)$, where the $\mathrm{F} 606 \mathrm{~W}_{\mathrm{HB}}$ values are taken from Dotter et al. (2010, Table 1). The histograms of the normalized $m_{\mathrm{F} 606 \mathrm{~W}}-m_{\mathrm{F} 814 \mathrm{~W}}$ color distribution for the HB and RGB sample are shown in the upper panel and colored blue and red, respectively. We have defined two points on the HB, $P_{\mathrm{A}}$ and $P_{\mathrm{B}}$, whose colors correspond to the fourth and the 96 percentile of the color distribution of HB stars. The color of the third point $P_{\mathrm{C}}$ is assumed as the median color of RGB stars; $L 1$ is defined as the color difference between $P_{\mathrm{C}}$ and $P_{\mathrm{B}}$ and $L 2$ as the color difference between $P_{\mathrm{B}}$ and $P_{\mathrm{A}}$. Uncertainties on $P_{\mathrm{A}}$, $P_{\mathrm{B}}, P_{\mathrm{C}}, L 1$, and $L 2$ are estimated for each GC by bootstrapping with replacements performed 1000 times on both the RGB and the HB. The error bars indicate one standard deviation $(68.27 \mathrm{th}$ percentile) of the bootstrapped measurements. The colors of $P_{\mathrm{A}}$, $P_{\mathrm{B}}, P_{\mathrm{C}}$, the values of $L 1$ and $L 2$, and the corresponding errors, are listed in Table 1.

RR Lyrae have been observed at random phases, and some of them could lie outside the instability strip. Similarly to what has been done in previous papers on the second parameter (e.g., Gratton et al. 2010; Dotter et al. 2010), we included in our analysis only those RR Lyrae that are close to the instability strip. In the Appendix we investigate the impact of excluding RR Lyrae that might be out of the instability strip in the determination of $L 1$ and $L 2$ and conclude that this does not affect the conclusions of our paper.

\section{GC PARAMETERS}

In the next section we shall compare the $L 1$ and $L 2 \mathrm{HB}$ morphology indicators with the physical and morphological GC parameters described here. Metallicity $([\mathrm{Fe} / \mathrm{H}])$, absolute visual magnitude $\left(M_{\mathrm{V}}\right)$, central velocity dispersion $\left(\sigma_{\mathrm{V}}\right)$, ellipticity $(\epsilon)$, central concentration $(c)$, core relaxation timescale $\left(\tau_{\mathrm{c}}\right)$, halfmass relaxation timescale $\left(\tau_{\mathrm{hm}}\right)$, logarithm of central stellar density $\left(\rho_{0}\right)$, central surface brightness $\left(\mu_{\mathrm{V}}\right)$, and Galactocentric distance $\left(R_{\mathrm{GC}}\right)$ are extracted from the 2010 edition of the Harris (1996) catalog. The specific frequency of RR Lyrae variables ( $\left.S_{\text {RR Lyrae }}\right)$ is taken from the 2003 edition of the Harris (1996) catalog. The fraction of binary stars have been measured by Milone et al. $(2012 \mathrm{~b}, 2012 \mathrm{c})$ in the core of the GC $\left(f_{\mathrm{bin}}^{\mathrm{C}}\right)$, in the region between the core and the half-mass radius $\left(f_{\mathrm{bin}}^{\mathrm{C}-\mathrm{HM}}\right)$, and outside the half-mass radius ( $f_{\text {bin }}^{\text {oHM }}$ ). We also use age and helium measurements, and some indicators of light-element variations, as discussed in detail in Sections 4.1 and 4.2, respectively.

\subsection{Age}

We adopt GC age measurements from De Angeli et al. (2005), Marín-Franch et al. (2009), VandenBerg et al. (2013), Leaman et al. (2013), and Dotter et al. (2010, 2011). De Angeli et al. (2005) determined ages for 55 GCs by measuring the difference between the $\mathrm{HB}$ and the turnoff in two internally photometrically homogeneous HST and ground-based databases (Piotto et al. 2002; Rosenberg et al. 1999). Among the GCs studied by De Angeli et al. (2005), 41 are in common with the ACS data.

Marín-Franch et al. (2009) used the same GO 10775 photometric database analyzed in the present paper to estimate relative ages for 64 GCs that are included in our data set with internal

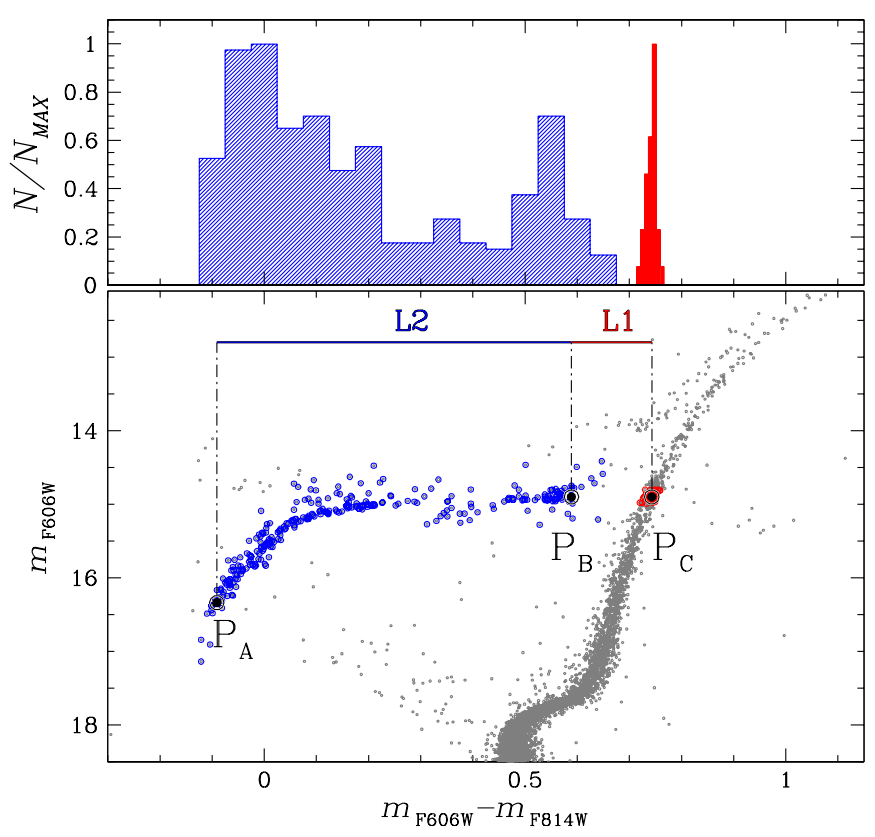

Figure 1. Upper panel: normalized histogram color distribution of stars in the HB sample (blue histogram) and RGB sample (red histogram) for NGC 5904. The RGB sample includes all the RGB stars with luminosity differing by less than \pm 0.1 F606W mag from the mean level of the HB. The two samples of HB and RGB stars are colored blue and red, respectively, in the lower-panel CMD, where we also show the points $P_{\mathrm{A}}, P_{\mathrm{B}}$, and $P_{\mathrm{C}}$ and the $L 1$ and $L 2$ segments (see the text for details).

(A color version of this figure is available in the online journal.)

uncertainties of $2 \%-7 \%$. Ages are derived by comparing the observed relative position of the GC main sequence turnoff (MSTO) with an isochrones-based grid of MSTOs.

More recently, VandenBerg et al. (2013) and Leaman et al. (2013) compared Victoria-Regina isochrones with photometry from Sarajedini et al. (2007) and Anderson et al. (2008) to derive ages for 61 GCs that are also included in our paper. To do this, they adopted an improved version of the classical "vertical method," which is based on the luminosity difference between the MSTO and the zero-age HB.

Age estimates by Dotter et al. $(2010,2011)$ are determined by using isochrone fitting to the CMDs for 59 of the GCs studied in this paper; note that these authors omitted six GCs present in the ACS Survey of Galactic GCs because of the presence of multiple stellar populations with either large helium variation or double subgiant branch (SGB). For the remainder of the ACS Survey GCs (NGC 1851, NGC 2808, NGC 6388, NGC 6441, NGC 6656, and NGC 6715), we calculated ages following the same recipes from Dotter and collaborators. We obtained $11.00 \pm 0.50$ Gyr (NGC 1851), $11.50 \pm 0.75 \mathrm{Gyr}$ (NGC 2808), $11.75 \pm 0.75$ Gyr (NGC 6388), $12.00 \pm 1.00 \mathrm{Gyr}$ (NGC 6441), $13.50 \pm 1.00$ Gyr (NGC 6656), and $13.25 \pm$ 0.75 Gyr (NGC 6715).

NGC 1851, NGC 6388, NGC 6656, and NGC 6715 each exhibit a double SGB (Milone et al. 2008; Marino et al. 2009; Piotto et al. 2012) that is consistent with two groups of stars that have either an age difference of 1-2 Gyr or almost the same age and different $\mathrm{C}+\mathrm{N}+\mathrm{O}$ abundance (Cassisi et al. 2008; Ventura et al. 2009). High-resolution spectroscopy of SGB and RGB stars in NGC 6656 showed that the faint SGB of this $\mathrm{GC}$ is indeed made of $\mathrm{C}+\mathrm{N}+\mathrm{O}$ enhanced stars (Marino et al. 2011a; Alves-Brito et al. 2012) and that by accounting for the chemical content of the two stellar groups, isochrone fitting 
Table 1

Horizontal-branch Parameters

\begin{tabular}{|c|c|c|c|c|c|c|}
\hline ID & $P_{\mathrm{A}}$ & $P_{\mathrm{B}}$ & $P_{\mathrm{C}}$ & $L 1$ & $L 2$ & Group \\
\hline AM-1 & $0.558 \pm 0.003$ & $0.627 \pm 0.003$ & $0.720 \pm 0.006$ & $0.093 \pm 0.007$ & $0.069 \pm 0.004$ & G2 \\
\hline ARP 2 & $0.105 \pm 0.013$ & $0.289 \pm 0.020$ & $0.780 \pm 0.003$ & $0.491 \pm 0.021$ & $0.184 \pm 0.021$ & G3 \\
\hline ERIDANUS & $0.584 \pm 0.011$ & $0.642 \pm 0.005$ & $0.755 \pm 0.011$ & $0.111 \pm 0.009$ & $0.058 \pm 0.012$ & G2 \\
\hline IC 4499 & $0.307 \pm 0.031$ & $0.815 \pm 0.024$ & $0.928 \pm 0.008$ & $0.113 \pm 0.026$ & $0.508 \pm 0.041$ & G2 \\
\hline LYNGA 7 & $1.387 \pm 0.008$ & $1.480 \pm 0.003$ & $1.534 \pm 0.004$ & $0.055 \pm 0.050$ & $0.093 \pm 0.009$ & G1 \\
\hline NGC 104 & $0.658 \pm 0.005$ & $0.726 \pm 0.004$ & $0.804 \pm 0.002$ & $0.078 \pm 0.005$ & $0.068 \pm 0.006$ & G1 \\
\hline NGC 288 & $-0.125 \pm 0.002$ & $0.212 \pm 0.086$ & $0.749 \pm 0.007$ & $0.534 \pm 0.086$ & $0.337 \pm 0.086$ & G3 \\
\hline NGC 362 & $0.049 \pm 0.063$ & $0.656 \pm 0.004$ & $0.742 \pm 0.002$ & $0.086 \pm 0.005$ & $0.608 \pm 0.064$ & $\mathrm{G} 2$ \\
\hline NGC 1261 & $-0.017 \pm 0.037$ & $0.628 \pm 0.003$ & $0.716 \pm 0.004$ & $0.088 \pm 0.005$ & $0.644 \pm 0.038$ & G2 \\
\hline NGC 1851 & $-0.018 \pm 0.010$ & $0.660 \pm 0.004$ & $0.758 \pm 0.002$ & $0.098 \pm 0.004$ & $0.679 \pm 0.010$ & G2 \\
\hline NGC 2298 & $0.150 \pm 0.014$ & $0.417 \pm 0.019$ & $0.902 \pm 0.007$ & $0.486 \pm 0.020$ & $0.267 \pm 0.023$ & G3 \\
\hline NGC 2419 & $-0.238 \pm 0.016$ & $0.614 \pm 0.047$ & $0.806 \pm 0.003$ & $0.192 \pm 0.046$ & $0.852 \pm 0.052$ & G2 \\
\hline NGC 2808 & $-0.094 \pm 0.010$ & $0.812 \pm 0.003$ & $0.906 \pm 0.001$ & $0.094 \pm 0.003$ & $0.904 \pm 0.010$ & G2 \\
\hline NGC 3201 & $0.231 \pm 0.021$ & $0.881 \pm 0.014$ & $0.986 \pm 0.007$ & $0.106 \pm 0.015$ & $0.649 \pm 0.022$ & $\mathrm{G} 2$ \\
\hline NGC 4147 & $-0.029 \pm 0.015$ & $0.447 \pm 0.026$ & $0.718 \pm 0.003$ & $0.271 \pm 0.027$ & $0.476 \pm 0.029$ & G2 \\
\hline NGC 4590 & $0.016 \pm 0.013$ & $0.541 \pm 0.029$ & $0.746 \pm 0.005$ & $0.205 \pm 0.029$ & $0.524 \pm 0.030$ & G2 \\
\hline NGC 4833 & $0.193 \pm 0.004$ & $0.731 \pm 0.037$ & $1.018 \pm 0.002$ & $0.287 \pm 0.037$ & $0.538 \pm 0.037$ & $\mathrm{G} 2$ \\
\hline NGC 5024 & $-0.049 \pm 0.008$ & $0.553 \pm 0.035$ & $0.711 \pm 0.002$ & $0.158 \pm 0.035$ & $0.602 \pm 0.036$ & G2 \\
\hline NGC 5053 & $-0.016 \pm 0.016$ & $0.483 \pm 0.009$ & $0.706 \pm 0.009$ & $0.223 \pm 0.090$ & $0.439 \pm 0.090$ & G2 \\
\hline NGC 5139 & $-0.158 \pm 0.003$ & $0.676 \pm 0.039$ & $0.842 \pm 0.002$ & $0.167 \pm 0.039$ & $0.834 \pm 0.039$ & G2 \\
\hline NGC 5272 & $-0.061 \pm 0.011$ & $0.552 \pm 0.016$ & $0.702 \pm 0.002$ & $0.150 \pm 0.016$ & $0.613 \pm 0.018$ & G2 \\
\hline NGC 5286 & $0.056 \pm 0.034$ & $0.726 \pm 0.034$ & $0.939 \pm 0.002$ & $0.213 \pm 0.034$ & $0.670 \pm 0.035$ & G2 \\
\hline NGC 5466 & $0.035 \pm 0.015$ & $0.492 \pm 0.061$ & $0.717 \pm 0.007$ & $0.225 \pm 0.062$ & $0.457 \pm 0.063$ & G2 \\
\hline NGC 5904 & $-0.088 \pm 0.008$ & $0.593 \pm 0.012$ & $0.743 \pm 0.002$ & $0.150 \pm 0.012$ & $0.681 \pm 0.014$ & G2 \\
\hline NGC 5927 & $1.108 \pm 0.003$ & $1.171 \pm 0.002$ & $1.214 \pm 0.003$ & $0.043 \pm 0.003$ & $0.062 \pm 0.004$ & G1 \\
\hline NGC 5986 & $0.080 \pm 0.013$ & $0.524 \pm 0.051$ & $0.984 \pm 0.003$ & $0.460 \pm 0.052$ & $0.443 \pm 0.053$ & G3 \\
\hline NGC 6093 & $-0.019 \pm 0.011$ & $0.401 \pm 0.059$ & $0.090 \pm 0.002$ & $0.464 \pm 0.059$ & $0.447 \pm 0.062$ & G3 \\
\hline NGC 6101 & $0.088 \pm 0.005$ & $0.311 \pm 0.024$ & $0.796 \pm 0.005$ & $0.485 \pm 0.025$ & $0.223 \pm 0.025$ & G3 \\
\hline NGC 6121 & $0.468 \pm 0.006$ & $1.037 \pm 0.019$ & $1.157 \pm 0.007$ & $0.120 \pm 0.020$ & $0.569 \pm 0.020$ & G2 \\
\hline NGC 6144 & $0.364 \pm 0.009$ & $0.593 \pm 0.023$ & $1.126 \pm 0.009$ & $0.533 \pm 0.024$ & $0.229 \pm 0.023$ & G3 \\
\hline NGC 6171 & $0.526 \pm 0.072$ & $1.039 \pm 0.014$ & $1.139 \pm 0.004$ & $0.100 \pm 0.014$ & $0.513 \pm 0.074$ & G2 \\
\hline NGC 6205 & $-0.243 \pm 0.003$ & $0.198 \pm 0.013$ & $0.725 \pm 0.002$ & $0.527 \pm 0.013$ & $0.441 \pm 0.012$ & G3 \\
\hline NGC 6218 & $0.042 \pm 0.009$ & $0.341 \pm 0.033$ & $0.902 \pm 0.004$ & $0.561 \pm 0.034$ & $0.299 \pm 0.035$ & G3 \\
\hline NGC 6254 & $0.120 \pm 0.005$ & $0.380 \pm 0.032$ & $0.968 \pm 0.003$ & $0.588 \pm 0.032$ & $0.260 \pm 0.033$ & G3 \\
\hline NGC 6304 & $1.164 \pm 0.003$ & $1.225 \pm 0.003$ & $1.287 \pm 0.005$ & $0.062 \pm 0.007$ & $0.060 \pm 0.004$ & G1 \\
\hline NGC 6341 & $-0.097 \pm 0.004$ & $0.445 \pm 0.074$ & $0.705 \pm 0.003$ & $0.261 \pm 0.075$ & $0.542 \pm 0.075$ & G2 \\
\hline NGC 6352 & $0.906 \pm 0.003$ & $0.962 \pm 0.002$ & $1.035 \pm 0.007$ & $0.072 \pm 0.007$ & $0.056 \pm 0.003$ & G1 \\
\hline NGC 6362 & $0.054 \pm 0.039$ & $0.675 \pm 0.002$ & $0.797 \pm 0.003$ & $0.122 \pm 0.004$ & $0.621 \pm 0.039$ & G2 \\
\hline NGC 6366 & $1.360 \pm 0.001$ & $1.436 \pm 0.005$ & $1.494 \pm 0.018$ & $0.076 \pm 0.005$ & $0.058 \pm 0.018$ & G1 \\
\hline NGC 6388 & $0.228 \pm 0.007$ & $1.064 \pm 0.002$ & $1.120 \pm 0.003$ & $0.057 \pm 0.004$ & $0.836 \pm 0.008$ & G1 \\
\hline NGC 6397 & $0.095 \pm 0.021$ & $0.327 \pm 0.023$ & $0.861 \pm 0.005$ & $0.534 \pm 0.023$ & $0.232 \pm 0.030$ & G3 \\
\hline NGC 6426 & $0.403 \pm 0.016$ & $0.922 \pm 0.018$ & $1.100 \pm 0.003$ & $0.178 \pm 0.018$ & $0.519 \pm 0.023$ & G2 \\
\hline NGC 6441 & $0.298 \pm 0.024$ & $1.202 \pm 0.001$ & $1.250 \pm 0.003$ & $0.048 \pm 0.003$ & $0.904 \pm 0.024$ & G1 \\
\hline NGC 6496 & $0.891 \pm 0.003$ & $0.946 \pm 0.004$ & $1.020 \pm 0.011$ & $0.074 \pm 0.011$ & $0.056 \pm 0.005$ & G1 \\
\hline NGC 6535 & $0.322 \pm 0.020$ & $0.593 \pm 0.022$ & $1.103 \pm 0.010$ & $0.510 \pm 0.026$ & $0.271 \pm 0.031$ & G3 \\
\hline NGC 6541 & $-0.104 \pm 0.026$ & $0.242 \pm 0.025$ & $0.805 \pm 0.003$ & $0.563 \pm 0.026$ & $0.347 \pm 0.033$ & G3 \\
\hline NGC 6584 & $0.097 \pm 0.024$ & $0.654 \pm 0.011$ & $0.756 \pm 0.003$ & $0.102 \pm 0.012$ & $0.558 \pm 0.026$ & G1 \\
\hline NGC 6624 & $0.892 \pm 0.004$ & $0.977 \pm 0.005$ & $1.005 \pm 0.004$ & $0.077 \pm 0.006$ & $0.085 \pm 0.006$ & G1 \\
\hline NGC 6637 & $0.786 \pm 0.004$ & $0.851 \pm 0.005$ & $0.928 \pm 0.002$ & $0.078 \pm 0.004$ & $0.065 \pm 0.005$ & G1 \\
\hline NGC 6652 & $0.733 \pm 0.008$ & $0.812 \pm 0.010$ & $0.885 \pm 0.004$ & $0.073 \pm 0.011$ & $0.080 \pm 0.012$ & G1 \\
\hline NGC 6656 & $0.135 \pm 0.010$ & $0.712 \pm 0.088$ & $1.048 \pm 0.027$ & $0.336 \pm 0.088$ & $0.577 \pm 0.087$ & $\mathrm{G} 2$ \\
\hline NGC 6681 & $-0.081 \pm 0.007$ & $0.253 \pm 0.045$ & $0.794 \pm 0.002$ & $0.558 \pm 0.046$ & $0.334 \pm 0.045$ & G3 \\
\hline NGC 6715 & $-0.140 \pm 0.003$ & $0.726 \pm 0.007$ & $0.860 \pm 0.001$ & $0.134 \pm 0.007$ & $0.866 \pm 0.007$ & G2 \\
\hline NGC 6717 & $0.111 \pm 0.010$ & $0.421 \pm 0.031$ & $0.916 \pm 0.007$ & $0.495 \pm 0.032$ & $0.310 \pm 0.033$ & G3 \\
\hline NGC 6723 & $-0.033 \pm 0.008$ & $0.671 \pm 0.006$ & $0.798 \pm 0.003$ & $0.127 \pm 0.007$ & $0.704 \pm 0.010$ & G2 \\
\hline NGC 6752 & $-0.211 \pm 0.006$ & $0.171 \pm 0.024$ & $0.749 \pm 0.002$ & $0.378 \pm 0.024$ & $0.578 \pm 0.025$ & G2 \\
\hline NGC 6779 & $0.134 \pm 0.007$ & $0.418 \pm 0.031$ & $0.926 \pm 0.005$ & $0.508 \pm 0.031$ & $0.284 \pm 0.030$ & G3 \\
\hline NGC 6809 & $0.014 \pm 0.012$ & $0.327 \pm 0.054$ & $0.803 \pm 0.004$ & $0.476 \pm 0.055$ & $0.313 \pm 0.053$ & G3 \\
\hline NGC 6838 & $0.863 \pm 0.006$ & $0.920 \pm 0.007$ & $1.003 \pm 0.010$ & $0.084 \pm 0.012$ & $0.057 \pm 0.010$ & G1 \\
\hline NGC 6934 & $0.039 \pm 0.010$ & $0.718 \pm 0.013$ & $0.815 \pm 0.003$ & $0.097 \pm 0.013$ & $0.678 \pm 0.016$ & G2 \\
\hline NGC 6981 & $0.415 \pm 0.011$ & $0.612 \pm 0.016$ & $0.754 \pm 0.003$ & $0.142 \pm 0.016$ & $0.570 \pm 0.019$ & $\mathrm{G} 2$ \\
\hline NGC 7006 & $0.089 \pm 0.009$ & $0.670 \pm 0.016$ & $0.793 \pm 0.007$ & $0.123 \pm 0.016$ & $0.581 \pm 0.018$ & G2 \\
\hline NGC 7078 & $-0.119 \pm 0.015$ & $0.594 \pm 0.011$ & $0.768 \pm 0.001$ & $0.174 \pm 0.011$ & $0.713 \pm 0.019$ & $\mathrm{G} 2$ \\
\hline NGC 7089 & $-0.189 \pm 0.013$ & $0.601 \pm 0.035$ & $0.751 \pm 0.001$ & $0.150 \pm 0.035$ & $0.790 \pm 0.037$ & G2 \\
\hline NGC 7099 & $-0.007 \pm 0.006$ & $0.253 \pm 0.102$ & $0.716 \pm 0.003$ & $0.462 \pm 0.103$ & $0.261 \pm 0.103$ & G3 \\
\hline PALOMAR 3 & $0.391 \pm 0.014$ & $0.545 \pm 0.016$ & $0.727 \pm 0.014$ & $0.182 \pm 0.030$ & $0.154 \pm 0.027$ & G2 \\
\hline
\end{tabular}


Table 1

(Continued)

\begin{tabular}{lcccccc}
\hline \hline ID & $P_{\mathrm{A}}$ & $P_{\mathrm{B}}$ & $P_{\mathrm{C}}$ & $L 1$ & $L 2$ & Group \\
\hline PALOMAR 4 & $0.544 \pm 0.007$ & $0.634 \pm 0.002$ & $0.737 \pm 0.007$ & $0.103 \pm 0.007$ & $0.090 \pm 0.007$ & $\mathrm{G} 2$ \\
PALOMAR 12 & $0.672 \pm 0.002$ & $0.693 \pm 0.003$ & $0.766 \pm 0.012$ & $0.069 \pm 0.012$ & $0.019 \pm 0.002$ & $\mathrm{G} 1$ \\
PALOMAR 14 & $0.595 \pm 0.009$ & $0.679 \pm 0.013$ & $0.762 \pm 0.026$ & $0.084 \pm 0.030$ & $0.083 \pm 0.015$ & $\mathrm{G} 2$ \\
PALOMAR 15 & $0.419 \pm 0.007$ & $0.617 \pm 0.037$ & $1.102 \pm 0.007$ & $0.485 \pm 0.038$ & $0.198 \pm 0.037$ & $\mathrm{G} 3$ \\
PYXIS & $0.827 \pm 0.008$ & $0.898 \pm 0.006$ & $0.964 \pm 0.006$ & $0.066 \pm 0.008$ & $0.071 \pm 0.010$ & $\mathrm{G} 2$ \\
RUPR 106 & $0.531 \pm 0.031$ & $0.741 \pm 0.008$ & $0.876 \pm 0.009$ & $0.135 \pm 0.012$ & $0.210 \pm 0.032$ & $\mathrm{G} 2$ \\
TERZAN 7 & $0.746 \pm 0.003$ & $0.778 \pm 0.004$ & $0.835 \pm 0.004$ & $0.057 \pm 0.007$ & $0.032 \pm 0.008$ & $\mathrm{G} 1$ \\
TERZAN 8 & $0.099 \pm 0.004$ & $0.322 \pm 0.050$ & $0.822 \pm 0.003$ & $0.500 \pm 0.050$ & $0.223 \pm 0.050$ & $\mathrm{G} 3$ \\
\hline
\end{tabular}

Table 2

Literature Estimate of the Maximum Helium Difference between Stellar Populations Based on the Analysis of Multiple MSs or Multiple RGBs

\begin{tabular}{lcc}
\hline \hline ID & $\Delta Y$ & Reference \\
\hline NGC 104 (47 Tuc) & $\sim 0.03$ & di Criscienzo et al. (2010); Milone et al. (2012d) \\
NGC 288 & $0.013 \pm 0.001$ & Piotto et al. (2013) \\
NGC 2419 & $\sim 0.17$ & di Criscienzo et al. (2011) \\
NGC 2808 & $\sim 0.14$ & Milone et al. (2012c) \\
NGC 5139 $(\omega$ Cen) & $0.14 \pm 0.01$ & King et al. (2012) \\
NGC 6397 & $\sim 0.01$ & Milone et al. (2012a) \\
NGC 6441 & $\sim 0.07$ & Bellini et al. (2013) \\
NGC 6752 & $0.04 \pm 0.01$ & Milone et al. (2013) \\
NGC 7078 & $0.053 \pm 0.015$ & A. P. Milone et al. (in preparation) \\
\hline
\end{tabular}

Notes. a Accurate analysis of the MS width by di Criscienzo et al. (2011) previously showed that any helium variation in NGC 6397 must be smaller than $\Delta Y \sim 0.02$.

of the double SGB indicates that their ages do not differ by more than $\sim 300$ Myr (Marino et al. 2012). Large star-to-star $\mathrm{C}+\mathrm{N}+\mathrm{O}$ variations, with faint $\mathrm{SGB}$-stars being also enhanced in $\mathrm{C}+\mathrm{N}+\mathrm{O}$, have been also observed in NGC 1851 (Yong et al. 2009 , and D. Yong et al. in preparation) even if this result is not confirmed by Villanova et al. (2010). For GCs with a double SGB, we assume the age obtained from fitting the bright SGB. To distinguish between GCs with single or bimodal SGBs, the statistical analysis of the relation between HB morphology and age presented in Section 5 will be presented with and without these double SGB GCs.

When investigating the effect of age on HB morphology, a challenge comes from the fact that the population of Milky Way GCs mainly consists of old objects. Young clusters are hence important in the present investigation as any effect of age on the HB morphology would be better identified when comparing clusters with large age differences. In order to better sample the Galactic GC population at all Galactocentric distances, Dotter et al. $(2010,2011)$ expanded the sample studied by MarínFranch et al. (2009) to include several more-distant GCs: AM-1 and Pal 14 (Dotter et al. 2008); Pal 3, Pal 4, and Eridanus (Stetson et al. 1999); and IC 4499, Pal 5, Pyxis, and Ruprecht 106 (Dotter et al. 2011). It turns out that several of them are also young clusters.

Ages from Dotter and collaborators are available for 73 GCs, but only 61, 41, and 61 of them are included in the age compilations published by Marín-Franch et al. (2009), by De Angeli et al. (2005), and by VandenBerg et al. (2013) and Leaman et al. (2013), respectively. Since the sample by Dotter and collaborators is the most complete and is based on the best data set available, we began by using their ages to investigate possible relations with $L 1$ and $L 2$. Although the other age compilations include a smaller GC sample, they can be used to demonstrate that our conclusions do not rely on a particular set of ages.
Another challenge comes from age errors that can be as large as 1.25 Gyr for the Dotter et al. (2010, 2011) sample. To minimize the impact of error measurements, our conclusions are based on the average properties of groups of GCs that will be defined in Section 5.1.

\subsection{Light-element and Helium Variations}

Our analysis makes use of several indicators of the intracluster light-element variations as well as of measurements of the helium differences among the multiple stellar populations in GCs. Some of these parameters have been defined several decades ago and are widely discussed in the context of the $2 \mathrm{ndP}$ phenomenon, while others have been introduced more recently.

Norris (1987) defined the ratio $\left(R_{\mathrm{CN}}\right)$ between $\mathrm{CN}$-strong and $\mathrm{CN}$-weak stars and provided $R_{\mathrm{CN}}$ measurements for a sample of $12 \mathrm{GCs}$, which was increased to 16 by Smith \& Mateo (1990). The interquartile range of the $[\mathrm{O} / \mathrm{Na}]$ ratio, $\mathrm{IQR}[\mathrm{O} / \mathrm{Na}]$, can be considered as another indicator of the internal light-element variation to quantify the extension of the sodium-oxygen anticorrelation (Carretta 2006). This parameter is available for 24 GCs: for 14 GCs, namely NGC 104, NGC 1904, NGC 2808, NGC 3201, NGC 4590, NGC 5904, NGC 6171, NGC 6218, NGC 6254, NGC 6388, NGC 6441, NGC 6809, NGC 6838, and NGC 7078, we used the IQR[O/Na] values listed by Carretta et al. (2010b). For the other 10 $\mathrm{GCs}$, we have calculated $\mathrm{IQR}[\mathrm{O} / \mathrm{Na}]$ by using oxygen and sodium abundances available in the literature. We obtained $\mathrm{IQR}[\mathrm{O} / \mathrm{Na}]=0.61$ and 0.67 for NGC 288 and NGC 362, respectively (Shetrone \& Keane 2000); IQR $[\mathrm{O} / \mathrm{Na}]=0.68$ for NGC 1851 (Villanova et al. 2010); IQR $[\mathrm{O} / \mathrm{Na}]=0.54$ and 1.04 for NGC 5272 and NGC 6205, respectively (Sneden et al. 2004); IQR $[\mathrm{O} / \mathrm{Na}]=0.28$ for NGC 6397 (Lind et al. 2011); $\mathrm{IQR}[\mathrm{O} / \mathrm{Na}]=1.16$ for NGC 6715 (Carretta et al. 2010a); $\mathrm{IQR}[\mathrm{O} / \mathrm{Na}]=0.91$ for NGC 6752 (Yong et al. 2008); and 
$\mathrm{IQR}[\mathrm{O} / \mathrm{Na}]=0.49,0.67$, and 1.07 for NGC 6121, NGC 6656, and NGC 5139, respectively (Marino et al. 2008, 2011b, 2011a).

In their survey of multiple stellar populations in GCs, Monelli et al. (2013) defined the photometric index $c_{\mathrm{UBI}}=$ $(U-B)-(B-I)$ and found that all the GCs they analyzed show a multimodal or spread RGB in the $V$ versus $c_{\text {UBI }}$ diagram, with the $c_{\text {UBI }}$ value of each star being related to its light-element abundances. The $c_{\mathrm{UBI}}$ index width of the RGB ( $\left.W_{\mathrm{RGB}}\right)$ is listed by Monelli et al. (2013) for 22 GCs.

Some stars in GCs have light-element abundance similar to halo-field stars of the same metallicity (e.g., Kraft 1994). It is widely accepted that these stars constitute the first stellar population and can be distinguished from the other GC stars either on the basis of their abundance of $\mathrm{C}, \mathrm{N}, \mathrm{Na}$, and $\mathrm{O}$ or their position in the CMD. For 47 Tuc, NGC 6397, NGC 6752, and NGC 288 we adopted the fraction of first-population stars with respect to the total population $\left(f_{\mathrm{POPI}}\right)$ determined from photometric studies (Milone et al. 2012c, 2012d, 2013; Piotto et al. 2013). For NGC 2808 and NGC 6121 we used the fractions derived by Marino et al. (2008) and Marino et al. (2014), and for NGC 3201, NGC 4590, NGC 5272, NGC 5904, NGC 6171, NGC 6205, NGC 6218, NGC 6254, NGC 6388, NGC 6496, NGC 6838, NGC 7078, and NGC 7079 we used the value given by Carretta et al. (2010b).

While the quantities $R_{\mathrm{CN}}, \mathrm{IQR}[\mathrm{O} / \mathrm{Na}]$, and $W_{\mathrm{RGB}}$ are mainly related to the internal variations of light elements, recent analyses based on multiwavelength photometry made it possible to estimate the helium difference between stellar populations in a single GC. These studies have revealed that the CMD of a GC is typically composed of intertwined sequences, the separate identities of which can be followed continuously from the MS up to the RGB. These sequences are associated with stellar populations with different light-element and helium abundances (e.g., Milone et al. 2012a, 2012d). The comparison of the observed multicolor difference between the different MSs and RGBs with colors obtained from appropriate theoretical stellar atmospheres provides an estimate of the maximum helium difference $(\Delta Y)$ between the stellar populations that is not based on HB stars. Although this technique has been applied to only a few GCs to date, we shall use the available $\Delta Y$ measurements, summarized in Table 2, to investigate possible relations with $\mathrm{HB}$ morphology in the next section.

While $\Delta Y$ indicates the maximum internal variation in helium, the $R$-parameter $(R)$ defined by Iben (1968) as the number ratio of HB to RGB stars brighter than the HB level is sensitive to the initial helium content of GCs. At a given metallicity, a higher initial He-content implies a brighter HB and, in turn, a lower number of RGB stars. In this paper we used the values of $R$ determined by Salaris et al. (2004). Gratton et al. (2010) introduced a similar parameter $R^{\prime}=N_{\mathrm{HB}} / N_{\mathrm{RGB}}^{\prime}$, where $N_{\mathrm{HB}}$ is the number of HB stars, $N_{\mathrm{RGB}}^{\prime}$ the number of RGB stars brighter than $V(\mathrm{HB})+1$, and $V(\mathrm{HB})$ is the $V$ magnitude of the HB taken from the Harris (1996) catalog. Gratton and collaborators used the $R$-parameter method (Iben 1968) to derive the He abundance for GCs $\left(Y\left(R^{\prime}\right)\right)$. As suggested by the referee, we extend our analysis to the $Y\left(R^{\prime}\right)$ values provided by Gratton et al. (2010).

\section{RELATIONSHIP BETWEEN HB-MORPHOLOGY AND GLOBULAR CLUSTER PARAMETERS}

In this section we investigate the correlations among $L 1$, $L 2$, and several physical and morphological parameters of their host GCs. Specifically, relations with metallicity, absolute magnitude, and age are discussed in Sections 5.1, 5.2, and 5.3, respectively. Section 5.4 investigates the correlations with the internal variations of the light elements and helium, while relations between $L 1$ and $L 2$ and the other parameters introduced in Section 4 are analyzed in Section 5.5.

When we compare two variables, as we do in the next section for $L 1, L 2$, and [Fe/H], we use the Spearman's rank correlation coefficient $r$ to estimate the statistical dependence between the two. Uncertainties in $r$ are estimated by means of bootstrapping statistics. We generated 1000 resamples of the observed data set, of equal size, and for each resample (1) (which is generated by random sampling with replacement from the original data set) we estimated $r_{\mathrm{i}}$. We considered the dispersion of the $r_{\mathrm{i}}$ measurements $\left(\sigma_{\mathrm{r}}\right)$ as indicative of the robustness of $r$ and provide the number of included GCs $(N)$.

\subsection{Metallicity}

The left panel of Figure 2 shows $L 1$ against the GC metallicity. An inspection of this plot reveals that all the metal-rich GCs have small $L 1$ values and, hence, red HBs. At lower metallicities, there are GCs with almost the same iron abundance and yet different $L 1$ values. ${ }^{11}$ This reflects the basic 2 ndP phenomenon. Indeed, if the all of the GCs followed the same relation in the $L 1$ versus $[\mathrm{Fe} / \mathrm{H}]$ plane, we would assume that metallicity alone is sufficient to determine $L 1$. The fact that we observe clusters with the same $[\mathrm{Fe} / \mathrm{H}]$ but different $L 1$ values indicates that apart from metallicity, at least one more parameter is at work.

Our finding that the analyzed GCs populate distinct regions in the $L 1$ versus $[\mathrm{Fe} / \mathrm{H}]$ plane and that the 2 ndP phenomenon is absent among the majority of metal-rich GCs motivated us to define three groups of GCs as follows:

1. The first group, G1, includes all the metal-rich GCs $([\mathrm{Fe} / \mathrm{H}] \geqslant-1.0)$.

2. The second, G2, is made of GCs with $[\mathrm{Fe} / \mathrm{H}]<-1.0$ and $L 1 \leqslant 0.4$.

3. The remaining GCs with $L 1>0.4$ belong to G3.

Since the 2 ndP phenomenon is absent among the majority of G1 GCs, we will also consider a group that includes all the GCs in $\mathrm{G} 2$ and G3, hereafter G2+G3. The statistical analysis presented in the following will be provided for all the GCs together, as well as for the different groups separately.

There is a significant anticorrelation between $L 1$ and $[\mathrm{Fe} / \mathrm{H}]$ among G2 GCs, with a Spearman's rank correlation coefficient $r_{\mathrm{G} 2}=-0.70\left(\sigma_{\mathrm{r}, \mathrm{G} 2}=0.08, N_{\mathrm{G} 2}=38\right)$ that drops to -0.88 if we consider G1 and G2 GCs together, an even stronger correlation. The Spearman's correlation coefficients and the corresponding $\sigma_{\mathrm{r}}$ values are listed in Table 3 for the groups of GCs defined above. There is no correlation between $L 2$ and $[\mathrm{Fe} / \mathrm{H}]$, either for G1 or G3 GCs.

Apart from NGC 6388 and NGC 6441, all G1 GCs host a purely red $\mathrm{HB}$ and have $L 2$ values smaller than the majority of the other GCs. In G2 and G3, GCs metallicity is not responsible for the extension of the $\mathrm{HB}(L 2)$ as shown in the right panel of Figure 2.

\subsection{Absolute Magnitude}

The left panel of Figure 3 shows that there is no significant correlation between $L 1$ and the GC absolute luminosity for

\footnotetext{
11 The fact that $L 1$ possesses a dependence on metallicity is expected from theory. Similarly, it is well known from theory that several other parameters may determine HB morphology and hence can affect the values of $L 1$ and $L 2$. This paper is an attempt to investigate those parameters that are actually at work by following a fully empirical approach.
} 

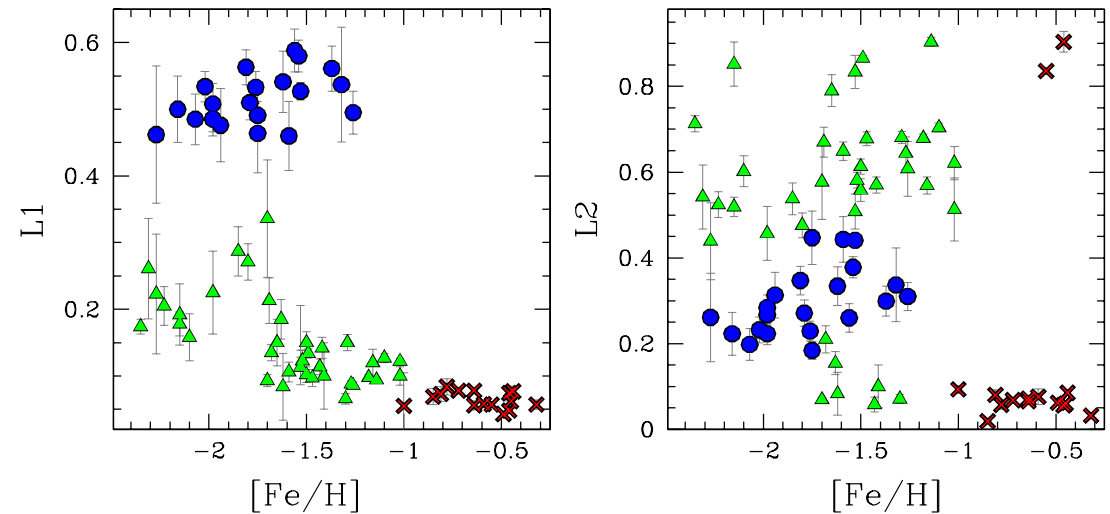

Figure 2. Left panel: $L 1$ vs. GC metallicity for the 72 GCs studied in this work. The G1, G2, and G3 GCs are colored red, green, and blue, respectively. Right panel: $L 2$ against metallicity.

(A color version of this figure is available in the online journal.)
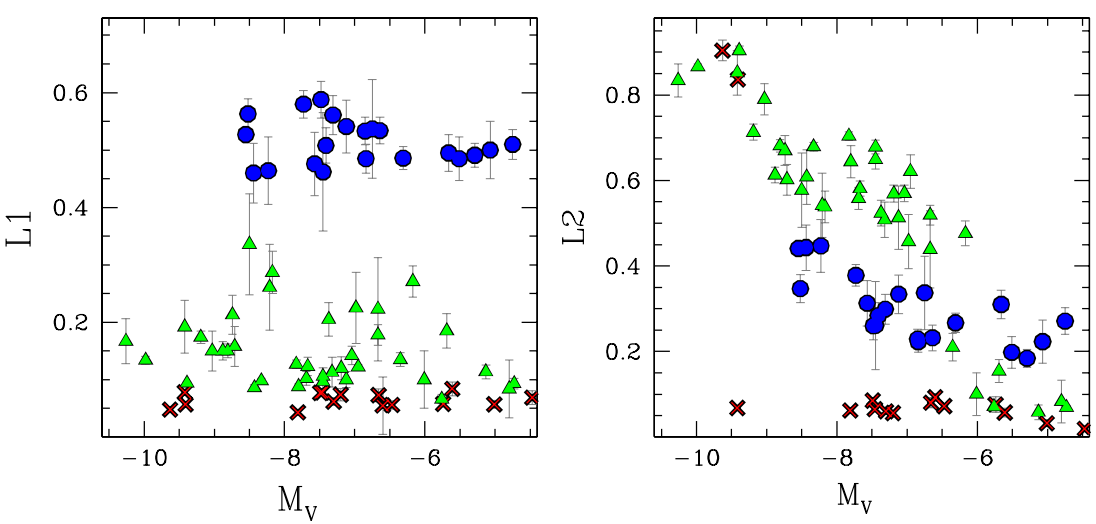

Figure 3. $L 1$ (left panel) and $L 2$ (right panel) as a function of GC absolute magnitude.

(A color version of this figure is available in the online journal.)

any of the groups of GCs defined above. In contrast, both G2 and G3 GCs exhibit significant anticorrelations between L2 and the absolute GC magnitude, which relates to the GC mass assuming all GCs have roughly the same mass-to-light ratio. This is shown in the right panel of Figure 3, where we plot $L 2$ as a function of $M_{\mathrm{V}}$. The Spearman's rank correlation coefficient is $r_{\mathrm{G} 2}=-0.89\left(\sigma_{\mathrm{r}, \mathrm{G} 2}=0.05, N=38\right)$ and $r_{\mathrm{G} 3}=$ $-0.71\left(\sigma_{\mathrm{r}, \mathrm{G} 3}=0.13, N_{\mathrm{G} 3}=21\right)$ for the $\mathrm{G} 2$ and $\mathrm{G} 3$ samples, respectively, $r_{\mathrm{G} 2+\mathrm{G} 3}=-0.80\left(\sigma_{\mathrm{r}, \mathrm{G} 2+\mathrm{G} 3}=0.06, N_{\mathrm{G} 2+\mathrm{G} 3}=59\right)$ for G2+G3 GCs.

\subsection{Age}

Histograms of the age distributions for G1, G2, and G3 GCs are shown in the upper panels of Figure 4 for the age measurements from Dotter et al. (2010, 2011; left panel) and Marín-Franch et al. (2009; right panel) and in Figure 5 for the age measurements of De Angeli et al. (2005; left panel) and VandenBerg et al. (2013) and Leaman et al. (2013; right panel). On average, G3 GCs are systematically older than G2 GCs, and this result is independent of the adopted age scale. Specifically, independently from the four adopted age scales, G2 GCs are, on average, younger than G3 ones by $\sim 1$ Gyr with G3 GCs clustered around the value of $\sim 13 \mathrm{Gyr}$ and G2 GCs spanning a wider age interval. The mean ages of G2 and G3 GCs are listed in Table 4.

The $L 1$ is plotted as a function of GC age in the middle panels of Figures 4 and 5. There is a positive correlation between age and $L 1$ for G2 GCs, with older G2 GCs having, on average, greater $L 1$ values. The Spearman's coefficient is high
$r_{\mathrm{G} 2} \geqslant 0.70$, except when we adopt ages from Marín-Franch et al. (2009), indicating that in the latter case the significance level is low. ${ }^{12}$

As a further check we have divided GCs into three subgroups with almost the same metallicity. We have defined a metal-poor $([\mathrm{Fe} / \mathrm{H}]<-1.7)$, a metal-intermediate $(-1.7<[\mathrm{Fe} / \mathrm{H}]<$ $-1.4)$, and a metal-rich $([\mathrm{Fe} / \mathrm{H}]>-1.4)$ group, and we investigate the age $-L 1$ relation for GCs in each of them. Results are listed in Table 4. In all the cases, G3 GCs are systematically older even if, especially for metal-poor GCs, the statistical significance of the measured age difference is marginal, but we are limited by small number statistics. The fact that G3 GCs are systematically older than G2 GCs and the presence of significant correlation between age and $L 1$ for G2 GCs indicate that GC age is partly responsible for the color distance between the RGB and the reddest part of the HB, with metallicity being the other parameter for $L 1$ extension. ${ }^{13}$

There is no significant correlation between $L 2$ and age as shown in the lower panels of Figures 4 and 5. GCs with a

\footnotetext{
12 As pointed out by the referee, there are three G3 GCs that, according to Marín-Franch et al. (2009), are younger than $~ 12.25$ Gyr. Their ages are in agreement with the average age of G3 GCs only to within 1.5-2.5\%. We are not able to say if this difference is due to measurement errors or is intrinsic. 13 Gratton et al. (2010) also derived ages for a subsample of the clusters studied in the present paper. To determine these ages, they used stellar masses derived from isochrones that include ages from Marín-Franch et al. (2009) and De Angeli et al. (2005) already analyzed in this paper. In addition, the way these ages are derived is closely related to the HB morphology. For these reasons we prefer to avoid using them in the context of this paper. Nevertheless, we verified that our conclusions remain unchanged when ages from Gratton et al. (2010) are used.
} 
Table 3

Spearman's Rank Correlation Coefficients Indicating the Statistical Dependence between L1 (Columns 2-5), L2 (Columns 6-9), and Several Parameters of the Host GCs for G1, G2, G3, and G2+G3 GCs

\begin{tabular}{|c|c|c|c|c|c|c|c|c|}
\hline \multirow[t]{2}{*}{ Parameter } & \multicolumn{3}{|c|}{$L 1$} & \multirow[b]{2}{*}{$\mathrm{G} 2+\mathrm{G} 3$} & \multicolumn{3}{|c|}{$L 2$} & \multirow[b]{2}{*}{$\mathrm{G} 2+\mathrm{G} 3$} \\
\hline & G1 & G2 & G3 & & G1 & G2 & G3 & \\
\hline$[\mathrm{Fe} / \mathrm{H}]$ & $\begin{array}{c}r=-0.13 \\
N=15 \\
\sigma_{\mathrm{r}}=0.26\end{array}$ & $\begin{array}{c}r=-0.70 \\
N=38 \\
\sigma_{\mathrm{r}}=0.08\end{array}$ & $\begin{array}{c}r=0.42 \\
N=21 \\
\sigma_{\mathrm{r}}=0.18\end{array}$ & $\begin{array}{c}r=-0.47 \\
N=59 \\
\sigma_{\mathrm{r}}=0.11\end{array}$ & $\begin{array}{c}r=-0.08 \\
N=15 \\
\sigma_{\mathrm{r}}=0.31\end{array}$ & $\begin{array}{c}r=0.19 \\
N=38 \\
\sigma_{\mathrm{r}}=0.16\end{array}$ & $\begin{array}{c}r=0.57 \\
N=21 \\
\sigma_{\mathrm{r}}=0.13\end{array}$ & $\begin{array}{c}r=0.24 \\
N=59 \\
\sigma_{\mathrm{r}}=0.13\end{array}$ \\
\hline$M_{\mathrm{V}}$ & $\begin{array}{c}r=0.09 \\
N=15 \\
\sigma_{\mathrm{r}}=0.13\end{array}$ & $\begin{array}{c}r=-0.23 \\
N=38 \\
\sigma_{\mathrm{r}}=0.16\end{array}$ & $\begin{array}{c}r=-0.08 \\
N=21 \\
\sigma_{\mathrm{r}}=0.24\end{array}$ & $\begin{array}{c}r=0.09 \\
N=59 \\
\sigma_{\mathrm{r}}=0.13\end{array}$ & $\begin{array}{c}r=-0.57 \\
N=15 \\
\sigma_{\mathrm{r}}=0.23\end{array}$ & $\begin{array}{c}r=-0.89 \\
N=38 \\
\sigma_{\mathrm{r}}=0.05\end{array}$ & $\begin{array}{c}r=-0.71 \\
N=21 \\
\sigma_{\mathrm{r}}=0.13\end{array}$ & $\begin{array}{c}r=-0.80 \\
N=59 \\
\sigma_{\mathrm{r}}=0.06\end{array}$ \\
\hline $\mathrm{AGE}_{\mathrm{D} 10}$ & $\begin{array}{c}r=0.35 \\
N=15 \\
\sigma_{\mathrm{r}}=0.20\end{array}$ & $\begin{array}{c}r=0.76 \\
N=37 \\
\sigma_{\mathrm{r}}=0.08\end{array}$ & $\begin{array}{c}r=-0.22 \\
N=21 \\
\sigma_{\mathrm{r}}=0.20\end{array}$ & $\begin{array}{c}r=0.72 \\
N=58 \\
\sigma_{\mathrm{r}}=0.08\end{array}$ & $\begin{array}{c}r=0.23 \\
N=15 \\
\sigma_{\mathrm{r}}=0.29\end{array}$ & $\begin{array}{c}r=0.28 \\
N=37 \\
\sigma_{\mathrm{r}}=0.18\end{array}$ & $\begin{array}{c}r=-0.07 \\
N=21 \\
\sigma_{\mathrm{r}}=0.24\end{array}$ & $\begin{array}{c}r=-0.06 \\
N=58 \\
\sigma_{\mathrm{r}}=0.16\end{array}$ \\
\hline $\mathrm{AGE}_{\mathrm{MF} 09}$ & $\begin{array}{c}r=0.38 \\
N=15 \\
\sigma_{\mathrm{r}}=0.26\end{array}$ & $\begin{array}{c}r=0.47 \\
N=26 \\
\sigma_{\mathrm{r}}=0.18\end{array}$ & $\begin{array}{c}r=-0.05 \\
N=20 \\
\sigma_{\mathrm{r}}=0.20\end{array}$ & $\begin{array}{c}r=0.38 \\
N=46 \\
\sigma_{\mathrm{r}}=0.15\end{array}$ & $\begin{array}{c}r=0.30 \\
N=15 \\
\sigma_{\mathrm{r}}=0.30\end{array}$ & $\begin{array}{c}r=-0.29 \\
N=26 \\
\sigma_{\mathrm{r}}=0.16\end{array}$ & $\begin{array}{c}r=0.17 \\
N=20 \\
\sigma_{\mathrm{r}}=0.22\end{array}$ & $\begin{array}{c}r=-0.30 \\
N=46 \\
\sigma_{\mathrm{r}}=0.14\end{array}$ \\
\hline $\mathrm{AGE}_{\mathrm{DA} 05}$ & $\begin{array}{c}r=0.60 \\
N=5 \\
\sigma_{\mathrm{r}}=0.37\end{array}$ & $\begin{array}{c}r=0.72 \\
N=25 \\
\sigma_{\mathrm{r}}=0.13\end{array}$ & $\begin{array}{c}r=-0.32 \\
N=11 \\
\sigma_{\mathrm{r}}=0.36\end{array}$ & $\begin{array}{c}r=0.57 \\
N=36 \\
\sigma_{\mathrm{r}}=0.13\end{array}$ & $\begin{array}{c}r=-0.60 \\
N=5 \\
\sigma_{\mathrm{r}}=0.40\end{array}$ & $\begin{array}{c}r=-0.50 \\
N=25 \\
\sigma_{\mathrm{r}}=0.14\end{array}$ & $\begin{array}{c}r=0.03 \\
N=11 \\
\sigma_{\mathrm{r}}=0.36\end{array}$ & $\begin{array}{c}r=-0.49 \\
N=36 \\
\sigma_{\mathrm{r}}=0.13\end{array}$ \\
\hline$A G E_{\mathrm{V} 13}$ & $\begin{array}{c}r=0.62 \\
N=12 \\
\sigma_{\mathrm{r}}=0.20\end{array}$ & $\begin{array}{c}r=0.73 \\
N=31 \\
\sigma_{\mathrm{r}}=0.09\end{array}$ & $\begin{array}{c}r=-0.21 \\
N=18 \\
\sigma_{\mathrm{r}}=0.25\end{array}$ & $\begin{array}{c}r=0.68 \\
N=49 \\
\sigma_{\mathrm{r}}=0.10\end{array}$ & $\begin{array}{c}r=0.62 \\
N=12 \\
\sigma_{\mathrm{r}}=0.21\end{array}$ & $\begin{array}{c}r=0.01 \\
N=31 \\
\sigma_{\mathrm{r}}=0.20\end{array}$ & $\begin{array}{c}r=-0.22 \\
N=18 \\
\sigma_{\mathrm{r}}=0.25\end{array}$ & $\begin{array}{c}r=-0.34 \\
N=49 \\
\sigma_{\mathrm{r}}=0.16\end{array}$ \\
\hline $\mathrm{AGE}_{\mathrm{D} 10}{ }^{\mathrm{a}}$ & $\begin{array}{c}r=0.35 \\
N=14 \\
\sigma_{\mathrm{r}}=0.20\end{array}$ & $\begin{array}{c}r=0.76 \\
N=34 \\
\sigma_{\mathrm{r}}=0.08\end{array}$ & $\begin{array}{c}r=-0.22 \\
N=21 \\
\sigma_{\mathrm{r}}=0.20\end{array}$ & $\begin{array}{c}r=0.74 \\
N=55 \\
\sigma_{\mathrm{r}}=0.07\end{array}$ & $\begin{array}{c}r=0.39 \\
N=14 \\
\sigma_{\mathrm{r}}=0.26\end{array}$ & $\begin{array}{c}r=0.30 \\
N=34 \\
\sigma_{\mathrm{r}}=0.19\end{array}$ & $\begin{array}{c}r=-0.08 \\
N=21 \\
\sigma_{\mathrm{r}}=0.24\end{array}$ & $\begin{array}{c}r=-0.08 \\
N=55 \\
\sigma_{\mathrm{r}}=0.17\end{array}$ \\
\hline $\mathrm{AGE}_{\mathrm{MF} 09^{\mathrm{a}}}$ & $\begin{array}{c}r=0.41 \\
N=14 \\
\sigma_{\mathrm{r}}=\end{array}$ & $\begin{array}{c}r=0.40 \\
N=23 \\
\sigma_{\mathrm{r}}=0.21\end{array}$ & $\begin{array}{c}r=-0.05 \\
N=20 \\
\sigma_{\mathrm{r}}=0.20\end{array}$ & $\begin{array}{c}r=0.33 \\
N=43 \\
\sigma_{\mathrm{r}}=0.16\end{array}$ & $\begin{array}{c}r=0.31 \\
N=14 \\
\sigma_{\mathrm{r}}=0.30\end{array}$ & $\begin{array}{c}r=-0.29 \\
N=23 \\
\sigma_{\mathrm{r}}=0.16\end{array}$ & $\begin{array}{c}r=0.17 \\
N=20 \\
\sigma_{\mathrm{r}}=0.22\end{array}$ & $\begin{array}{c}r=-0.30 \\
N=43 \\
\sigma_{\mathrm{r}}=0.14\end{array}$ \\
\hline $\mathrm{AGE}_{\mathrm{DA} 05}{ }^{\mathrm{a}}$ & $\begin{array}{c}r=0.60 \\
N=5 \\
\sigma_{\mathrm{r}}=0.37\end{array}$ & $\begin{array}{c}r=0.71 \\
N=23 \\
\sigma_{\mathrm{r}}=0.14\end{array}$ & $\begin{array}{c}r=-0.32 \\
N=11 \\
\sigma_{\mathrm{r}}=0.36\end{array}$ & $\begin{array}{c}r=0.53 \\
N=34 \\
\sigma_{\mathrm{r}}=0.15\end{array}$ & $\begin{array}{c}r=-0.60 \\
N=5 \\
\sigma_{\mathrm{r}}=0.40\end{array}$ & $\begin{array}{c}r=-0.53 \\
N=23 \\
\sigma_{\mathrm{r}}=0.14\end{array}$ & $\begin{array}{c}r=0.03 \\
N=11 \\
\sigma_{\mathrm{r}}=0.36\end{array}$ & $\begin{array}{c}r=-0.47 \\
N=34 \\
\sigma_{\mathrm{r}}=0.13\end{array}$ \\
\hline $\mathrm{AGE}_{\mathrm{V} 13}{ }^{\mathrm{a}}$ & $\begin{array}{c}r=0.62 \\
N=12 \\
\sigma_{\mathrm{r}}=0.20\end{array}$ & $\begin{array}{c}r=0.70 \\
N=28 \\
\sigma_{\mathrm{r}}=0.10\end{array}$ & $\begin{array}{c}r=-0.21 \\
N=18 \\
\sigma_{\mathrm{r}}=0.25\end{array}$ & $\begin{array}{c}r=0.65 \\
N=46 \\
\sigma_{\mathrm{r}}=0.10\end{array}$ & $\begin{array}{c}r=0.62 \\
N=12 \\
\sigma_{\mathrm{r}}=0.21\end{array}$ & $\begin{array}{c}r=0.07 \\
N=28 \\
\sigma_{\mathrm{r}}=0.22\end{array}$ & $\begin{array}{c}r=-0.22 \\
N=18 \\
\sigma_{\mathrm{r}}=0.25\end{array}$ & $\begin{array}{c}r=-0.32 \\
N=46 \\
\sigma_{\mathrm{r}}=0.16\end{array}$ \\
\hline $\log (\Delta Y)$ & $\begin{array}{c}- \\
N=2 \\
-\end{array}$ & $\begin{array}{c}r=-0.30 \\
N=5 \\
\sigma_{\mathrm{r}}=0.54\end{array}$ & $\begin{array}{c}- \\
N=2 \\
-\end{array}$ & $\begin{array}{c}r=-0.71 \\
N=7 \\
\sigma_{\mathrm{r}}=0.31\end{array}$ & $\begin{array}{c}- \\
N=2 \\
-\end{array}$ & $\begin{array}{c}r=0.70 \\
N=5 \\
\sigma_{\mathrm{r}}=0.37\end{array}$ & $\begin{array}{c}- \\
N=2 \\
-\end{array}$ & $\begin{array}{c}r=0.89 \\
N=7 \\
\sigma_{\mathrm{r}}=0.17\end{array}$ \\
\hline$\sigma_{\mathrm{V}}$ & $\begin{array}{c}r=-0.54 \\
N=6 \\
\sigma_{\mathrm{r}}=0.38\end{array}$ & $\begin{array}{c}r=-0.07 \\
N=7 \\
\sigma_{\mathrm{r}}=0.22\end{array}$ & $\begin{array}{c}r=0.10 \\
N=13 \\
\sigma_{\mathrm{r}}=0.30\end{array}$ & $\begin{array}{c}r=-0.02 \\
N=38 \\
\sigma_{\mathrm{r}}=0.15\end{array}$ & $\begin{array}{c}r=0.66 \\
N=6 \\
\sigma_{\mathrm{r}}=0.29\end{array}$ & $\begin{array}{c}r=0.79 \\
N=7 \\
\sigma_{\mathrm{r}}=0.12\end{array}$ & $\begin{array}{c}r=0.40 \\
N=13 \\
\sigma_{\mathrm{r}}=0.28\end{array}$ & $\begin{array}{c}r=0.46 \\
N=38 \\
\sigma_{\mathrm{r}}=0.14\end{array}$ \\
\hline $\mathrm{c}$ & $\begin{array}{c}r=0.13 \\
N=14 \\
\sigma_{\mathrm{r}}=0.25\end{array}$ & $\begin{array}{c}r=0.08 \\
N=34 \\
\sigma_{\mathrm{r}}=0.17\end{array}$ & $\begin{array}{c}r=0.26 \\
N=16 \\
\sigma_{\mathrm{r}}=0.23\end{array}$ & $\begin{array}{c}r=-0.10 \\
N=50 \\
\sigma_{\mathrm{r}}=0.14\end{array}$ & $\begin{array}{c}r=0.07 \\
N=14 \\
\sigma_{\mathrm{r}}=0.31\end{array}$ & $\begin{array}{c}r=0.54 \\
N=34 \\
\sigma_{\mathrm{r}}=0.14\end{array}$ & $\begin{array}{c}r=0.52 \\
N=16 \\
\sigma_{\mathrm{r}}=0.23\end{array}$ & $\begin{array}{c}r=0.55 \\
N=50 \\
\sigma_{\mathrm{r}}=0.10\end{array}$ \\
\hline$\mu_{\mathrm{V}}$ & $\begin{array}{c}r=-0.01 \\
N=14 \\
\sigma_{\mathrm{r}}=0.28\end{array}$ & $\begin{array}{c}r=-0.07 \\
N=37 \\
\sigma_{\mathrm{r}}=0.18\end{array}$ & $\begin{array}{c}r=-0.23 \\
N=21 \\
\sigma_{\mathrm{r}}=0.23\end{array}$ & $\begin{array}{c}r=-0.07 \\
N=58 \\
\sigma_{\mathrm{r}}=0.14\end{array}$ & $\begin{array}{c}r=-0.65 \\
N=14 \\
\sigma_{\mathrm{r}}=0.21\end{array}$ & $\begin{array}{c}r=-0.76 \\
N=37 \\
\sigma_{\mathrm{r}}=0.10\end{array}$ & $\begin{array}{c}r=-0.68 \\
N=21 \\
\sigma_{\mathrm{r}}=0.15\end{array}$ & $\begin{array}{c}r=-0.57 \\
N=58 \\
\sigma_{\mathrm{r}}=0.11\end{array}$ \\
\hline$\rho$ & $\begin{array}{c}r=-0.09 \\
N=14 \\
\sigma_{\mathrm{r}}=0.26\end{array}$ & $\begin{array}{c}r=0.05 \\
N=37 \\
\sigma_{\mathrm{r}}=0.18\end{array}$ & $\begin{array}{c}r=0.29 \\
N=21 \\
\sigma_{\mathrm{r}}=0.21\end{array}$ & $\begin{array}{c}r=0.14 \\
N=58 \\
\sigma_{\mathrm{r}}=0.14\end{array}$ & $\begin{array}{c}r=0.69 \\
N=14 \\
\sigma_{\mathrm{r}}=0.18\end{array}$ & $\begin{array}{c}r=0.69 \\
N=37 \\
\sigma_{\mathrm{r}}=0.12\end{array}$ & $\begin{array}{c}r=0.56 \\
N=21 \\
\sigma_{\mathrm{r}}=0.19\end{array}$ & $\begin{array}{c}r=0.47 \\
N=58 \\
\sigma_{\mathrm{r}}=0.13\end{array}$ \\
\hline $\log \left(\tau_{\mathrm{c}}\right)$ & $\begin{array}{c}r=-0.31 \\
N=14 \\
\sigma_{\mathrm{r}}=0.21\end{array}$ & $\begin{array}{c}r=0.09 \\
N=37 \\
\sigma_{\mathrm{r}}=0.18\end{array}$ & $\begin{array}{c}r=-0.28 \\
N=21 \\
\sigma_{\mathrm{r}}=0.20\end{array}$ & $\begin{array}{c}r=-0.21 \\
N=58 \\
\sigma_{\mathrm{r}}=0.13\end{array}$ & $\begin{array}{c}r=-0.15 \\
N=14 \\
\sigma_{\mathrm{r}}=0.32\end{array}$ & $\begin{array}{c}r=-0.35 \\
N=37 \\
\sigma_{\mathrm{r}}=0.18\end{array}$ & $\begin{array}{c}r=-0.8 \\
N=21 \\
\sigma_{\mathrm{r}}=0.23\end{array}$ & $\begin{array}{c}r=-0.20 \\
N=58 \\
\sigma_{\mathrm{r}}=0.15\end{array}$ \\
\hline $\log \left(\tau_{\mathrm{h}}\right)$ & $\begin{array}{c}r=-0.29 \\
N=15 \\
\sigma_{\mathrm{r}}=0.28\end{array}$ & $\begin{array}{c}r=0.19 \\
N=37 \\
\sigma_{\mathrm{r}}=0.18\end{array}$ & $\begin{array}{c}r=-0.21 \\
N=21 \\
\sigma_{\mathrm{r}}=0.18\end{array}$ & $\begin{array}{c}r=-0.33 \\
N=58 \\
\sigma_{\mathrm{r}}=0.13\end{array}$ & $\begin{array}{c}r=-0.28 \\
N=15 \\
\sigma_{\mathrm{r}}=0.27\end{array}$ & $\begin{array}{c}r=-0.13 \\
N=37 \\
\sigma_{\mathrm{r}}=0.18\end{array}$ & $\begin{array}{c}r=-0.23 \\
N=21 \\
\sigma_{\mathrm{r}}=0.24\end{array}$ & $\begin{array}{c}r=0.08 \\
N=58 \\
\sigma_{\mathrm{r}}=0.15\end{array}$ \\
\hline$\epsilon$ & $\begin{array}{c}r=-0.10 \\
N=12 \\
\sigma_{\mathrm{r}}=0.32\end{array}$ & $\begin{array}{c}r=0.45 \\
N=30 \\
\sigma_{\mathrm{r}}=0.14\end{array}$ & $\begin{array}{c}r=0.14 \\
N=17 \\
\sigma_{\mathrm{r}}=0.27\end{array}$ & $\begin{array}{c}r=0.07 \\
N=47 \\
\sigma_{\mathrm{r}}=0.15\end{array}$ & $\begin{array}{c}r=0.05 \\
N=12 \\
\sigma_{\mathrm{r}}=0.33\end{array}$ & $\begin{array}{c}r=-0.05 \\
N=30 \\
\sigma_{\mathrm{r}}=0.20\end{array}$ & $\begin{array}{c}r=-0.16 \\
N=17 \\
\sigma_{\mathrm{r}}=0.27\end{array}$ & $\begin{array}{c}r=0.09 \\
N=47 \\
\sigma_{\mathrm{r}}=0.15\end{array}$ \\
\hline $\log \left(R_{\mathrm{GC}}[\mathrm{kpc})\right.$ & $\begin{array}{c}r=-0.08 \\
N=15 \\
\sigma_{\mathrm{r}}=0.27\end{array}$ & $\begin{array}{c}r=-0.18 \\
N=38 \\
\sigma_{\mathrm{r}}=0.16\end{array}$ & $\begin{array}{c}r=-0.29 \\
N=21 \\
\sigma_{\mathrm{r}}=0.19\end{array}$ & $\begin{array}{c}r=-0.45 \\
N=59 \\
\sigma_{\mathrm{r}}=0.11\end{array}$ & $\begin{array}{c}r=-0.50 \\
N=15 \\
\sigma_{\mathrm{r}}=0.22\end{array}$ & $\begin{array}{c}r=-0.44 \\
N=38 \\
\sigma_{\mathrm{r}}=0.16\end{array}$ & $\begin{array}{c}r=-0.50 \\
N=21 \\
\sigma_{\mathrm{r}}=0.19\end{array}$ & $\begin{array}{c}r=-0.17 \\
N=59 \\
\sigma_{\mathrm{r}}=0.15\end{array}$ \\
\hline
\end{tabular}


Table 3

(Continued)

\begin{tabular}{|c|c|c|c|c|c|c|c|c|}
\hline \multirow[t]{2}{*}{ Parameter } & \multicolumn{3}{|c|}{$L 1$} & \multirow[b]{2}{*}{$\mathrm{G} 2+\mathrm{G} 3$} & \multicolumn{3}{|c|}{$L 2$} & \multirow[b]{2}{*}{$\mathrm{G} 2+\mathrm{G} 3$} \\
\hline & G1 & G2 & G3 & & G1 & $\mathrm{G} 2$ & G3 & \\
\hline$S_{\text {RR Lyrae }}$ & $\begin{array}{c}r=-0.01 \\
N=14 \\
\sigma_{\mathrm{r}}=0.27\end{array}$ & $\begin{array}{c}r=-0.26 \\
N=36 \\
\sigma_{\mathrm{r}}=0.17\end{array}$ & $\begin{array}{c}r=-0.48 \\
N=20 \\
\sigma_{\mathrm{r}}=0.17\end{array}$ & $\begin{array}{c}r=-0.66 \\
N=56 \\
\sigma_{\mathrm{r}}=0.11\end{array}$ & $\begin{array}{c}r=-0.12 \\
N=14 \\
\sigma_{\mathrm{r}}=0.22\end{array}$ & $\begin{array}{c}r=-0.68 \\
N=36 \\
\sigma_{\mathrm{r}}=0.19\end{array}$ & $\begin{array}{c}r=-0.51 \\
N=20 \\
\sigma_{\mathrm{r}}=0.24\end{array}$ & $\begin{array}{c}r=-0.32 \\
N=56 \\
\sigma_{\mathrm{r}}=0.13\end{array}$ \\
\hline$f_{\text {bin }}^{\mathrm{C}}$ & $\begin{array}{c}r=0.19 \\
N=9 \\
\sigma_{\mathrm{r}}=0.40\end{array}$ & $\begin{array}{c}r=-0.03 \\
N=16 \\
\sigma_{\mathrm{r}}=0.30\end{array}$ & $\begin{array}{c}r=-0.22 \\
N=15 \\
\sigma_{\mathrm{r}}=0.26\end{array}$ & $\begin{array}{c}r=-0.18 \\
N=31 \\
\sigma_{\mathrm{r}}=0.18\end{array}$ & $\begin{array}{c}r=-0.12 \\
N=9 \\
\sigma_{\mathrm{r}}=0.36\end{array}$ & $\begin{array}{c}r=-0.68 \\
N=16 \\
\sigma_{\mathrm{r}}=0.15\end{array}$ & $\begin{array}{c}r=-0.51 \\
N=15 \\
\sigma_{\mathrm{r}}=0.25\end{array}$ & $\begin{array}{c}r=-0.32 \\
N=31 \\
\sigma_{\mathrm{r}}=0.17\end{array}$ \\
\hline$f_{\text {bin }}^{\mathrm{C}-\mathrm{HM}}$ & $\begin{array}{c}r=-0.09 \\
N=11 \\
\sigma_{\mathrm{r}}=0.35\end{array}$ & $\begin{array}{c}r=-0.19 \\
N=24 \\
\sigma_{\mathrm{r}}=0.19\end{array}$ & $\begin{array}{c}r=-0.18 \\
N=16 \\
\sigma_{\mathrm{r}}=0.29\end{array}$ & $\begin{array}{c}r=-0.15 \\
N=40 \\
\sigma_{\mathrm{r}}=0.14\end{array}$ & $\begin{array}{c}r=-0.67 \\
N=11 \\
\sigma_{\mathrm{r}}=0.23\end{array}$ & $\begin{array}{c}r=-0.47 \\
N=24 \\
\sigma_{\mathrm{r}}=0.20\end{array}$ & $\begin{array}{c}r=-0.56 \\
N=16 \\
\sigma_{\mathrm{r}}=0.23\end{array}$ & $\begin{array}{c}r=-0.27 \\
N=40 \\
\sigma_{\mathrm{r}}=0.15\end{array}$ \\
\hline$f_{\text {bin }}^{\mathrm{oHM}}$ & $\begin{array}{c}r=0.49 \\
N=11 \\
\sigma_{\mathrm{r}}=0.29\end{array}$ & $\begin{array}{c}r=-0.14 \\
N=18 \\
\sigma_{\mathrm{r}}=0.24\end{array}$ & $\begin{array}{c}r=-0.07 \\
N=17 \\
\sigma_{\mathrm{r}}=0.28\end{array}$ & $\begin{array}{c}r=-0.02 \\
N=35 \\
\sigma_{\mathrm{r}}=0.17\end{array}$ & $\begin{array}{c}r=-0.75 \\
N=11 \\
\sigma_{\mathrm{r}}=0.18\end{array}$ & $\begin{array}{c}r=-0.45 \\
N=18 \\
\sigma_{\mathrm{r}}=0.21\end{array}$ & $\begin{array}{c}r=-0.69 \\
N=17 \\
\sigma_{\mathrm{r}}=0.19\end{array}$ & $\begin{array}{c}r=-0.39 \\
N=35 \\
\sigma_{\mathrm{r}}=0.15 \\
\end{array}$ \\
\hline$W_{\mathrm{RGB}}$ & $\begin{array}{c}- \\
N=3 \\
-\end{array}$ & $\begin{array}{c}r=-0.45 \\
N=9 \\
\sigma_{\mathrm{r}}=0.31\end{array}$ & $\begin{array}{c}r=-0.08 \\
N=9 \\
\sigma_{\mathrm{r}}=0.35\end{array}$ & $\begin{array}{c}r=-0.23 \\
N=18 \\
\sigma_{\mathrm{r}}=0.22\end{array}$ & $\begin{array}{c}- \\
N=3 \\
-\end{array}$ & $\begin{array}{c}r=0.50 \\
N=9 \\
\sigma_{\mathrm{r}}=0.35\end{array}$ & $\begin{array}{c}r=0.35 \\
N=9 \\
\sigma_{\mathrm{r}}=0.37\end{array}$ & $\begin{array}{c}r=0.30 \\
N=18 \\
\sigma_{\mathrm{r}}=0.23 \\
\end{array}$ \\
\hline $\mathrm{IQR}([\mathrm{O} / \mathrm{Na}])$ & $\begin{array}{c}- \\
N=4 \\
-\end{array}$ & $\begin{array}{c}r=-0.13 \\
N=13 \\
\sigma_{\mathrm{r}}=0.26\end{array}$ & $\begin{array}{c}r=0.00 \\
N=8 \\
\sigma_{\mathrm{r}}=0.38\end{array}$ & $\begin{array}{c}r=-0.02 \\
N=21 \\
\sigma_{\mathrm{r}}=0.21\end{array}$ & $\begin{array}{c}- \\
N=4 \\
-\end{array}$ & $\begin{array}{c}r=0.82 \\
N=13 \\
\sigma_{\mathrm{r}}=0.13\end{array}$ & $\begin{array}{c}r=0.90 \\
N=8 \\
\sigma_{\mathrm{r}}=0.17\end{array}$ & $\begin{array}{c}r=0.41 \\
N=21 \\
\sigma_{\mathrm{r}}=0.21\end{array}$ \\
\hline$R_{\mathrm{CN}}$ & $\begin{array}{c}- \\
N=2 \\
-\end{array}$ & $\begin{array}{c}r=0.29 \\
N=11 \\
\sigma_{\mathrm{r}}=0.30\end{array}$ & $\begin{array}{c}- \\
N=3 \\
-\end{array}$ & $\begin{array}{c}r=0.13 \\
N=14 \\
\sigma_{\mathrm{r}}=0.27\end{array}$ & $\begin{array}{c}- \\
N=2 \\
-\end{array}$ & $\begin{array}{c}r=0.47 \\
N=11 \\
\sigma_{\mathrm{r}}=0.24\end{array}$ & $\begin{array}{c}- \\
N=3 \\
-\end{array}$ & $\begin{array}{c}r=0.38 \\
N=14 \\
\sigma_{\mathrm{r}}=0.27\end{array}$ \\
\hline$R$ & $\begin{array}{c}r=0.07 \\
N=8 \\
\sigma_{\mathrm{r}}=0.40\end{array}$ & $\begin{array}{c}r=0.17 \\
N=19 \\
\sigma_{\mathrm{r}}=0.26\end{array}$ & $\begin{array}{c}r=-0.07 \\
N=7 \\
\sigma_{\mathrm{r}}=0.39\end{array}$ & $\begin{array}{c}r=0.11 \\
N=26 \\
\sigma_{\mathrm{r}}=0.19\end{array}$ & $\begin{array}{c}r=0.26 \\
N=8 \\
\sigma_{\mathrm{r}}=0.35\end{array}$ & $\begin{array}{c}r=0.19 \\
N=19 \\
\sigma_{\mathrm{r}}=0.24\end{array}$ & $\begin{array}{c}r=-0.32 \\
N=7 \\
\sigma_{\mathrm{r}}=0.42\end{array}$ & $\begin{array}{c}r=0.07 \\
N=26 \\
\sigma_{\mathrm{r}}=0.22\end{array}$ \\
\hline$f_{\text {POPI }}$ & $\begin{array}{c}- \\
N=4 \\
-\end{array}$ & $\begin{array}{c}r=0.38 \\
N=8 \\
\sigma_{\mathrm{r}}=0.39\end{array}$ & $\begin{array}{c}r=-0.32 \\
N=7 \\
\sigma_{\mathrm{r}}=0.36\end{array}$ & $\begin{array}{c}r=-0.03 \\
N=15 \\
\sigma_{\mathrm{r}}=0.29\end{array}$ & $\begin{array}{c}- \\
N=4 \\
-\end{array}$ & $\begin{array}{c}r=-0.12 \\
N=8 \\
\sigma_{\mathrm{r}}=0.38\end{array}$ & $\begin{array}{c}r=-0.07 \\
N=7 \\
\sigma_{\mathrm{r}}=0.36\end{array}$ & $\begin{array}{c}r=-0.02 \\
N=15 \\
\sigma_{\mathrm{r}}=0.27\end{array}$ \\
\hline $\mathrm{Y}\left(\mathrm{R}^{\prime}\right)$ & $\begin{array}{c}r=-0.27 \\
N=11 \\
\sigma_{\mathrm{r}}=0.36\end{array}$ & $\begin{array}{c}r=-0.16 \\
N=25 \\
\sigma_{\mathrm{r}}=0.23\end{array}$ & $\begin{array}{c}r=0.12 \\
N=17 \\
\sigma_{\mathrm{r}}=0.28\end{array}$ & $\begin{array}{c}r=0.07 \\
N=42 \\
\sigma_{\mathrm{r}}=0.15\end{array}$ & $\begin{array}{c}r=0.15 \\
N=11 \\
\sigma_{\mathrm{r}}=0.34\end{array}$ & $\begin{array}{c}r=0.44 \\
N=25 \\
\sigma_{\mathrm{r}}=0.21\end{array}$ & $\begin{array}{c}r=-0.06 \\
N=17 \\
\sigma_{\mathrm{r}}=0.26\end{array}$ & $\begin{array}{c}r=0.04 \\
N=42 \\
\sigma_{\mathrm{r}}=0.16\end{array}$ \\
\hline$L 2$ & $\begin{array}{c}r=-0.35 \\
N=15 \\
\sigma_{\mathrm{r}}=0.24\end{array}$ & $\begin{array}{c}r=0.05 \\
N=38 \\
\sigma_{\mathrm{r}}=0.17\end{array}$ & $\begin{array}{c}r=0.10 \\
N=21 \\
\sigma_{\mathrm{r}}=0.25\end{array}$ & $\begin{array}{c}r=-0.35 \\
N=59 \\
\sigma_{\mathrm{r}}=0.15\end{array}$ & $\begin{array}{c}r=1.00 \\
N=15 \\
\sigma_{\mathrm{r}}=0.00\end{array}$ & $\begin{array}{c}r=1.00 \\
N=38 \\
\sigma_{\mathrm{r}}=0.00\end{array}$ & $\begin{array}{c}r=1.00 \\
N=21 \\
\sigma_{\mathrm{r}}=0.00\end{array}$ & $\begin{array}{c}r=1.00 \\
N=59 \\
\sigma_{\mathrm{r}}=0.00\end{array}$ \\
\hline HBR & $\begin{array}{c}r=0.43 \\
N=13 \\
\sigma_{\mathrm{r}}=0.28\end{array}$ & $\begin{array}{c}r=0.79 \\
N=37 \\
\sigma_{\mathrm{r}}=0.07\end{array}$ & $\begin{array}{c}r=0.48 \\
N=21 \\
\sigma_{\mathrm{r}}=0.16\end{array}$ & $\begin{array}{c}r=0.91 \\
N=58 \\
\sigma_{\mathrm{r}}=0.02\end{array}$ & $\begin{array}{c}r=-0.24 \\
N=13 \\
\sigma_{\mathrm{r}}=0.27\end{array}$ & $\begin{array}{c}r=0.44 \\
N=37 \\
\sigma_{\mathrm{r}}=0.16\end{array}$ & $\begin{array}{c}r=-0.04 \\
N=21 \\
\sigma_{\mathrm{r}}=0.26\end{array}$ & $\begin{array}{c}r=-0.15 \\
N=58 \\
\sigma_{\mathrm{r}}=0.17\end{array}$ \\
\hline$\delta M$ & $\begin{array}{c}r=-0.48 \\
N=12 \\
\sigma_{\mathrm{r}}=0.24\end{array}$ & $\begin{array}{c}r=-0.36 \\
N=25 \\
\sigma_{\mathrm{r}}=0.20\end{array}$ & $\begin{array}{c}r=0.12 \\
N=17 \\
\sigma_{\mathrm{r}}=0.29\end{array}$ & $\begin{array}{c}r=-0.19 \\
N=42 \\
\sigma_{\mathrm{r}}=0.30\end{array}$ & $\begin{array}{c}r=0.39 \\
N=12 \\
\sigma_{\mathrm{r}}=0.30\end{array}$ & $\begin{array}{c}r=0.66 \\
N=25 \\
\sigma_{\mathrm{r}}=0.14\end{array}$ & $\begin{array}{c}r=0.80 \\
N=17 \\
\sigma_{\mathrm{r}}=0.15\end{array}$ & $\begin{array}{c}r=0.48 \\
N=42 \\
\sigma_{\mathrm{r}}=0.13 \\
\end{array}$ \\
\hline$\Delta M_{\text {median }}$ & $\begin{array}{c}r=-0.47 \\
N=11 \\
\sigma_{\mathrm{r}}=0.28\end{array}$ & $\begin{array}{c}r=-0.68 \\
N=24 \\
\sigma_{\mathrm{r}}=0.10\end{array}$ & $\begin{array}{c}r=0.50 \\
N=17 \\
\sigma_{\mathrm{r}}=0.19\end{array}$ & $\begin{array}{c}r=0.00 \\
N=41 \\
\sigma_{\mathrm{r}}=0.17\end{array}$ & $\begin{array}{c}r=0.39 \\
N=11 \\
\sigma_{\mathrm{r}}=0.30\end{array}$ & $\begin{array}{c}r=0.63 \\
N=24 \\
\sigma_{\mathrm{r}}=0.17\end{array}$ & $\begin{array}{c}r=0.35 \\
N=17 \\
\sigma_{\mathrm{r}}=0.22\end{array}$ & $\begin{array}{c}r=0.14 \\
N=41 \\
\sigma_{\mathrm{r}}=0.17\end{array}$ \\
\hline$L_{\mathrm{t}}$ & $\begin{array}{c}- \\
N=4 \\
-\end{array}$ & $\begin{array}{c}r=-0.21 \\
N=23 \\
\sigma_{\mathrm{r}}=0.19\end{array}$ & $\begin{array}{c}r=0.68 \\
N=11 \\
\sigma_{\mathrm{r}}=0.21\end{array}$ & $\begin{array}{c}r=-0.06 \\
N=34 \\
\sigma_{\mathrm{r}}=0.19\end{array}$ & $\begin{array}{c}- \\
N=4 \\
-\end{array}$ & $\begin{array}{c}r=0.62 \\
N=23 \\
\sigma_{\mathrm{r}}=0.17\end{array}$ & $\begin{array}{c}r=0.38 \\
N=11 \\
\sigma_{\mathrm{r}}=0.33\end{array}$ & $\begin{array}{c}r=0.44 \\
N=34 \\
\sigma_{\mathrm{r}}=0.18\end{array}$ \\
\hline $\log \left(T_{\text {eff,MAX }}\right)$ & $\begin{array}{c}r=0.10 \\
N=8 \\
\sigma_{\mathrm{r}}=0.44\end{array}$ & $\begin{array}{c}r=0.10 \\
N=17 \\
\sigma_{\mathrm{r}}=0.25\end{array}$ & $\begin{array}{c}r=-0.14 \\
N=8 \\
\sigma_{\mathrm{r}}=0.34\end{array}$ & $\begin{array}{c}r=0.27 \\
N=25 \\
\sigma_{\mathrm{r}}=0.20\end{array}$ & $\begin{array}{c}r=0.71 \\
N=8 \\
\sigma_{\mathrm{r}}=0.26\end{array}$ & $\begin{array}{c}r=0.77 \\
N=17 \\
\sigma_{\mathrm{r}}=0.16\end{array}$ & $\begin{array}{c}r=0.90 \\
N=8 \\
\sigma_{\mathrm{r}}=0.14\end{array}$ & $\begin{array}{c}r=0.20 \\
N=25 \\
\sigma_{\mathrm{r}}=0.21\end{array}$ \\
\hline$\Delta(V-I)$ & $\begin{array}{c}r=0.21 \\
N=15 \\
\sigma_{\mathrm{r}}=0.26\end{array}$ & $\begin{array}{c}r=0.66 \\
N=38 \\
\sigma_{\mathrm{r}}=0.12\end{array}$ & $\begin{array}{c}r=0.64 \\
N=21 \\
\sigma_{\mathrm{r}}=0.14\end{array}$ & $\begin{array}{c}r=0.76 \\
N=59 \\
\sigma_{\mathrm{r}}=0.08\end{array}$ & $\begin{array}{c}r=0.49 \\
N=15 \\
\sigma_{\mathrm{r}}=0.23\end{array}$ & $\begin{array}{c}r=0.57 \\
N=38 \\
\sigma_{\mathrm{r}}=0.14\end{array}$ & $\begin{array}{c}r=0.75 \\
N=21 \\
\sigma_{\mathrm{r}}=0.14\end{array}$ & $\begin{array}{c}r=0.10 \\
N=59 \\
\sigma_{\mathrm{r}}=0.14\end{array}$ \\
\hline
\end{tabular}

Notes. The values of $\sigma_{\mathrm{r}}$, which provide an estimate of the robustness of $r$ measurements, and the numbers of analyzed GCs $(N)$ are also listed. ${ }^{a}$ GCs with double SGB excluded from the analysis. 

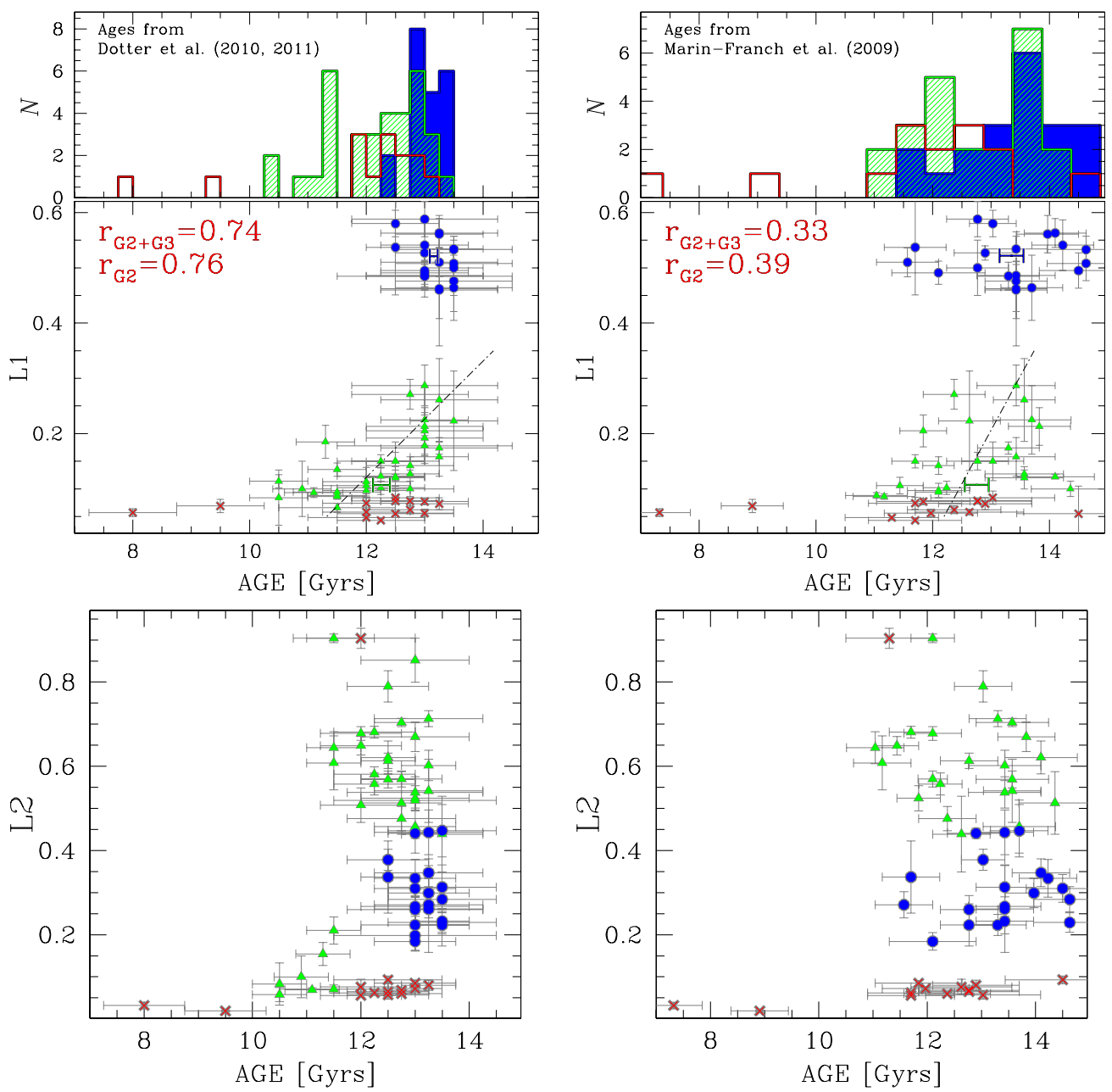

Figure 4. $L 2$ (lower panels) and $L 1$ (middle panels) against age. The histograms of age distribution for the G1 (red), G2 (green), and G3 (blue) GCs are plotted in the upper panel. In the left and right panels we used age measurements from Dotter et al. (2010, 2011) and Marín-Franch et al. (2009), respectively. Black dash-dotted lines in the middle panel are the best-fitting straight lines for the G2 sample. The Spearman's coefficients $r_{\mathrm{G} 2}$ and $r_{\mathrm{G} 2+\mathrm{G} 3}$ are also indicated.

(A color version of this figure is available in the online journal.)

double or multimodal SGB, namely NGC 1851, NGC 6388, NGC 6656, and NGC 6715, have been excluded from the statistical analysis above. As discussed in Section 4.1, the large fraction of faint-SGB stars observed in these GCs can affect the age measurements. For completeness we provide in Table 3 the values of the Spearman's rank correlation coefficients to estimate the statistical significance of $L 1, L 2$, and age correlations, together with results for the whole sample of GCs. The main results of this section remain unchanged when the GCs above are included in the analysis.

\subsection{Helium and Light Elements}

As mentioned in Section 1, the recent findings that in some GCs, groups of stars with different light-element abundances populate different HB segments strongly suggest that certain aspects of HB morphology may be strictly connected with multiple populations. To further investigate this scenario, in Figures 6-8 we show the relations between $L 1$ and $L 2$ and those quantities indicating intracluster chemical variations, which we introduced in Section 4.2. As shown in Figures 6 and 7, there is no significant correlation between $L 1$ and $W_{\mathrm{RGB}}, \mathrm{IQR}([\mathrm{O} / \mathrm{Na}])$, $R_{\mathrm{CN}}$, or $f_{\mathrm{POPI}}$ and no correlation between $L 2$ and $W_{\mathrm{RGB}}$ or $f_{\mathrm{POPI}}$. No significant correlations are observed between $L 1$ and $R$ or $Y\left(R^{\prime}\right)$ or between $L 2$ and $R$ or $Y\left(R^{\prime}\right)$. A mild correlation between
$L 2$ and $Y\left(R^{\prime}\right)$ cannot be ruled out for G2 GCs $\left(r_{\mathrm{G} 2}=0.44\right.$, $\left.\sigma_{r, \mathrm{G} 2}=0.21\right)$.

Figure 7 also shows that, on average, GCs with extended HBs have more extended $\mathrm{Na}-\mathrm{O}$ anticorrelations, as demonstrated by the significant correlation between $L 2$ and $\mathrm{IQR}([\mathrm{O} / \mathrm{Na}])$ obtained for GCs in both G2 and G3. This result confirms the findings by Carretta et al. (2007) and Gratton et al. (2010). Among G2 GCs, those with large $\mathrm{CN}$-strong and $\mathrm{CN}$-weak populations $\left(R_{\mathrm{CN}}>2\right)$ have, on average, a more extended HB. The small number of G2 and G3 GCs where $R_{\mathrm{CN}}$ measurements are available prevents us from making any strong conclusion regarding the significance of the correlation with $L 1$ and $L 2$.

Theoretical models predict that star-to-star light-element variations observed in GC stars are associated with helium differences that lead to HB stars with different masses because of the well-known inverse relationship between helium abundance and stellar mass for fixed metallicity and age (e.g., Ventura \& D'Antona 2005, and references therein). ${ }^{14}$ The relation between

\footnotetext{
14 The helium content of a star affects its location along the HB as follows. When compared with helium-normal stars $(Y \sim 0.25)$ of the same age and metallicity, He-enhanced stars have shorter main sequence lifetimes and hence smaller masses at the main sequence turnoff for a fixed age. Assuming the He-normal and He-enhanced stars lose a similar amount of mass on the RGB, the He-enriched stars will have a smaller $\mathrm{H}$-rich envelope on the $\mathrm{HB}$ and thus populate a hotter, bluer portion of the HB than the He-normal stars (e.g., Cassisi et al. 2012).
} 

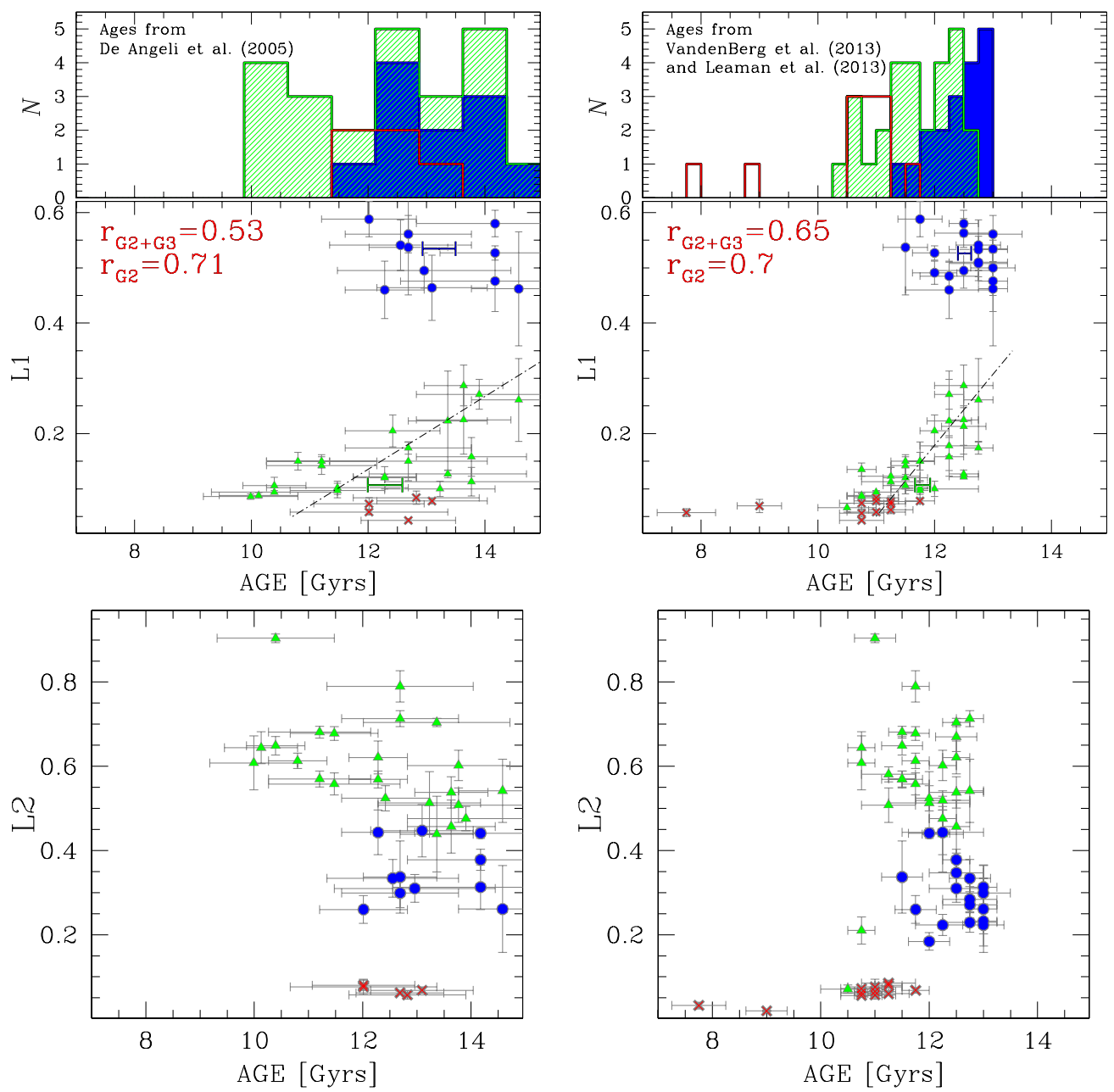

Figure 5. Same as in Figure 4 but for age measurements from De Angeli et al. (2005; left panels) and for ages derived by VandenBerg et al. (2013) and Leaman et al. (2013; right panels).

(A color version of this figure is available in the online journal.)

Table 4

Average Ages for G2 and G3 GCs

\begin{tabular}{lcc}
\hline \hline Author & $\begin{array}{c}\text { Age }_{\mathrm{G} 2} \\
(\mathrm{Gyr})\end{array}$ & $\begin{array}{c}\text { Age }_{\mathrm{G} 3} \\
(\mathrm{Gyr})\end{array}$ \\
\hline Dotter et al. (2010, 2011) & All \\
Marín-Franch et al. (2009) & $12.3 \pm 0.1$ & $13.2 \pm 0.1$ \\
De Angeli et al. (2005) & $12.8 \pm 0.2$ & $13.4 \pm 0.2$ \\
VandenBerg et al. (2013) and Leaman et al. (2013) & $12.3 \pm 0.3$ & $13.2 \pm 0.3$ \\
\hline & $11.8 \pm 0.1$ & $12.5 \pm 0.1$ \\
\hline Dotter et al. (2010, 2011) & Metal-rich sample \\
Marín-Franch et al. (2009) & $12.1 \pm 0.2$ \\
De Angeli et al. (2005) & $12.7 \pm 0.3$ & $12.9 \pm 0.3$ \\
VandenBerg et al. (2013) and Leaman et al. (2013) & $11.6 \pm 0.5$ & $13.3 \pm 0.1$ \\
\hline & $11.4 \pm 0.2$ & $12.8 \pm 0.1$ \\
\hline Dotter et al. (2010, 2011) & Metal-intermediate sample \\
Marín-Franch et al. (2009) & $11.9 \pm 0.2$ \\
De Angeli et al. (2005) & $12.5 \pm 0.3$ & $13.0 \pm 0.1$ \\
VandenBerg et al. (2013) and Leaman et al. (2013) & $11.7 \pm 0.5$ & $13.3 \pm 0.3$ \\
& $11.6 \pm 0.1$ & $13.0 \pm 0.5$ \\
\hline & Metal-poor sample \\
\hline Dotter et al. (2010, 2011) & $13.1 \pm 0.1$ & $12.2 \pm 0.2$ \\
Marín-Franch et al. (2009) & $13.0 \pm 0.3$ & $13.3 \pm 0.1$ \\
De Angeli et al. (2005) & $13.5 \pm 0.3$ & $14.0 \pm 0.5$ \\
VandenBerg et al. (2013) and Leaman et al. (2013) & $12.4 \pm 0.1$ & $12.7 \pm 0.1$ \\
\hline
\end{tabular}



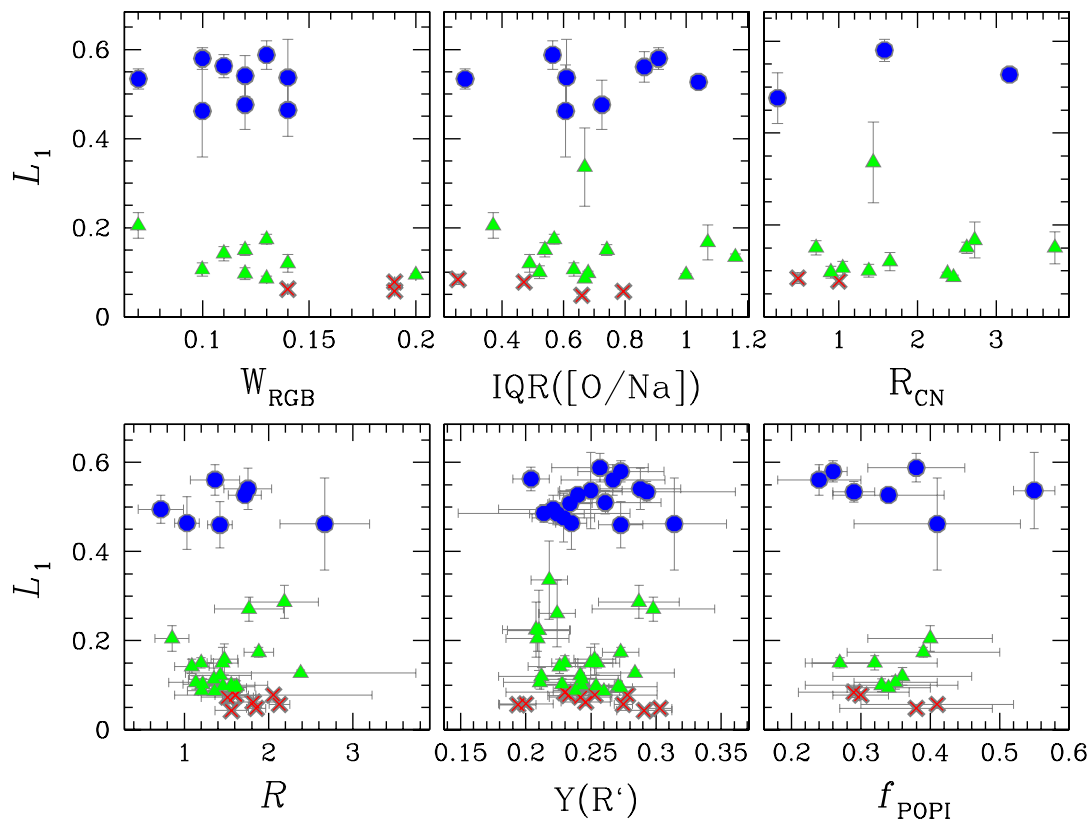

Figure 6. Upper panels: $L 1$ as a function of the width of the RGB in the $c_{\mathrm{UBI}}$ index (left), the interquartile range of the [O/Na] ratio (middle), and the ratio between $\mathrm{CN}$-strong and $\mathrm{CN}$-weak stars (right). Lower panels: $L 1$ as a function of the R-parameter, which is the number ratio of HB to RGB stars brighter than the HB level (left); the helium abundance inferred by Gratton et al. (2010) from the Iben (1968) method based on the $R$-parameter (middle); and the fraction of first-population stars (right).

(A color version of this figure is available in the online journal.)
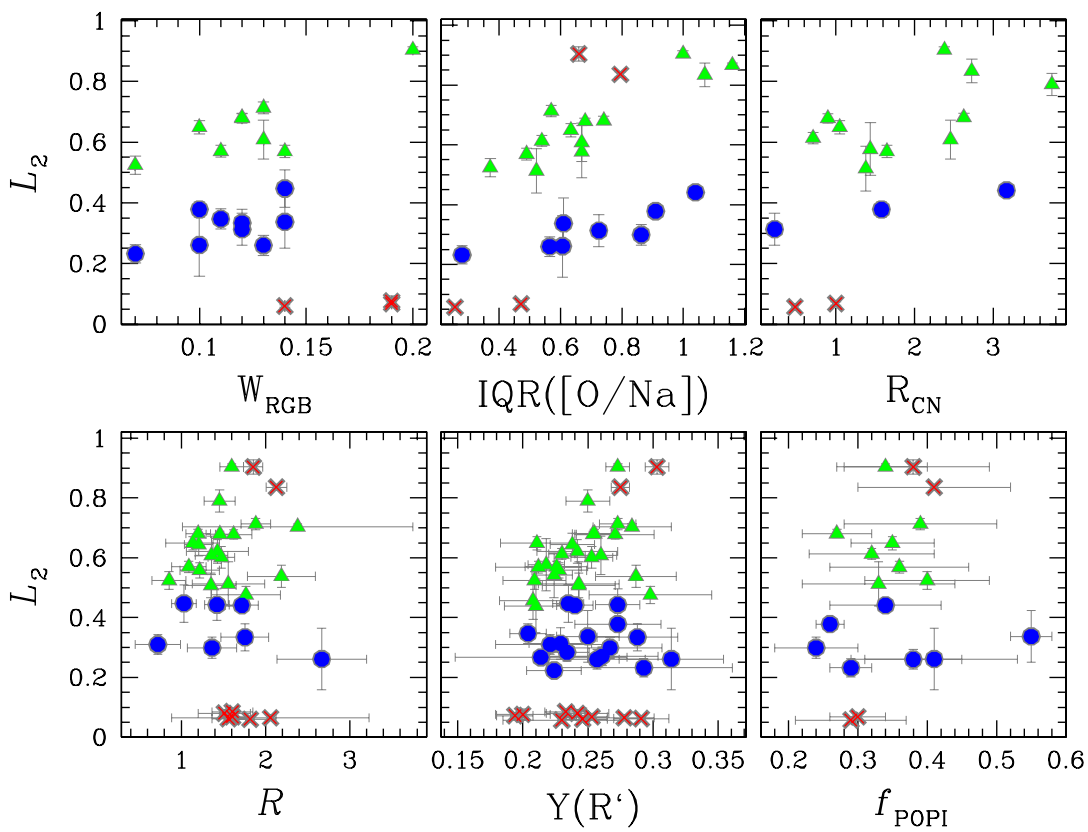

Figure 7. Same as in Figure 6 but for $L 2$.

(A color version of this figure is available in the online journal.)

$L 1, L 2$, and the maximum internal helium difference measured from MS studies is plotted in Figure 8. The tight correlation between $L 2\left(r_{\mathrm{G} 2+\mathrm{G} 3}=0.89, \sigma_{\mathrm{r}, \mathrm{G} 2+\mathrm{G} 3}=0.17, N_{\mathrm{G} 2+\mathrm{G} 3}=7\right)$ and the small corresponding value of $\sigma_{\mathrm{r}}$ for $\mathrm{G} 2+\mathrm{G} 3$ clusters confirms theoretical indications that helium-enhanced stellar population are responsible of the HB extension.

\subsection{Relationships with Other Parameters of the Host Globular Clusters}

Figure 9 shows other monovariate relations involving $L 1$. There is no significant correlation between $L 1$ and central velocity dispersion, King (1962) model central concentration, central brightness, central density, core and half mass relaxation time, GC ellipticity, Galactocentric distance, and binary fraction. This is confirmed by the values of the Spearman's correlation coefficient listed in Table 3.

In Figure 10 we see that $L 2$ correlates with $\rho$ and $\sigma_{\mathrm{V}}$ for G2 and G3 GCs and anticorrelates with $\mu_{\mathrm{V}}$ and $f_{\text {bin }}^{\mathrm{C}, \mathrm{C}-\mathrm{HM}, \mathrm{oHM}}$ for each group of GCs, even if the anticorrelation is less or not significant for the G2+G3 sample. These results are not unexpected as these quantities also correlate with GC mass (Djorgovski \& Meylan 1994). 

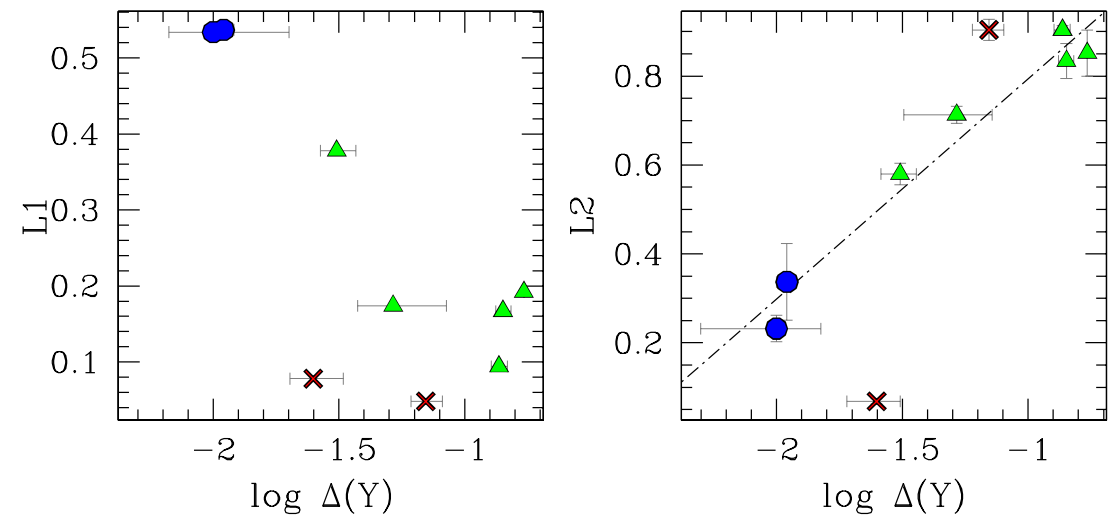

Figure 8. $L 1$ (left panel) and $L 2$ (right panel) as a function of the logarithm of the maximum helium difference among stellar populations in GCs. The black line is the best-fitting straight line for G2+G3 GCs.

(A color version of this figure is available in the online journal.)
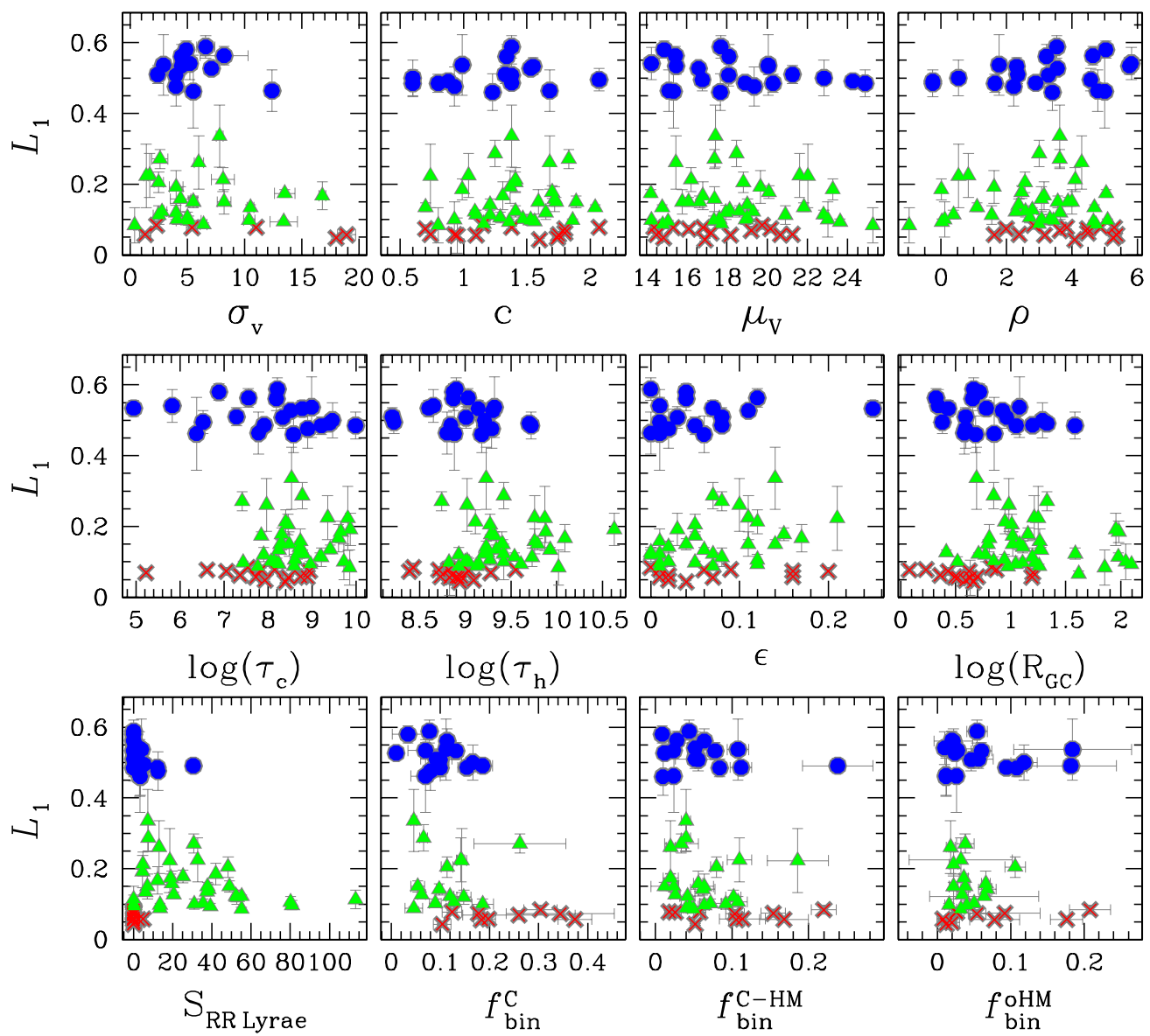

Figure 9. $L 1$ as a function of some parameters of the host GCs. From left to right: central velocity dispersion, King (1962) model central concentration, central luminosity brightness, and central luminosity density (top), core and half mass relaxation time, GC ellipticity, and logarithm of Galactocentric distance (middle), frequency of binaries, and fraction of binaries in the core, in the region between the core and the half-mass radius, and outside the half-mass radius (bottom). $\sigma_{\mathrm{v}}$ is given in $\mathrm{km} \mathrm{s}^{-1}, \mu_{\mathrm{V}}$ in $V$ magnitude per square arcsecond, $\tau_{\mathrm{c}}$ and $\tau_{\mathrm{h}}$ in years, and $R_{\mathrm{GC}}$ in kpc.

(A color version of this figure is available in the online journal.)

\section{COMPARISON WITH THE LITERATURE}

According to Gratton et al. (2010), in the ADS database there are more than 200 papers dedicated to the $2 \mathrm{ndP}$ phenomenon. Hence, any comparison here with the wide literature on the HB 2ndP can only be very far from complete. In this section we discuss some of the more relevant results. We refer the reader to review papers (e.g., Freeman \& Norris 1981; Catelan 2009) and references therein for a complete view on this topic.
As already mentioned in Section 1, works on HB morphology in GCs make use of different HB metrics. In Figures 11 and 12 , we compare $L 1$ and $L 2$ with other quantities used to parameterize HB morphology. The parameter to describe HB morphology that is mostly used in literature is the HB Type index or HBR (see Section 1). Figures 11 and 12 compare $L 1$ and $L 2$ with HBR. There is a linear correlation between $L 1$ and HBR for G2 GCs, and then HBR saturates for G1 and for G3 GCs; $L 2$ does not correlate with HBR. 

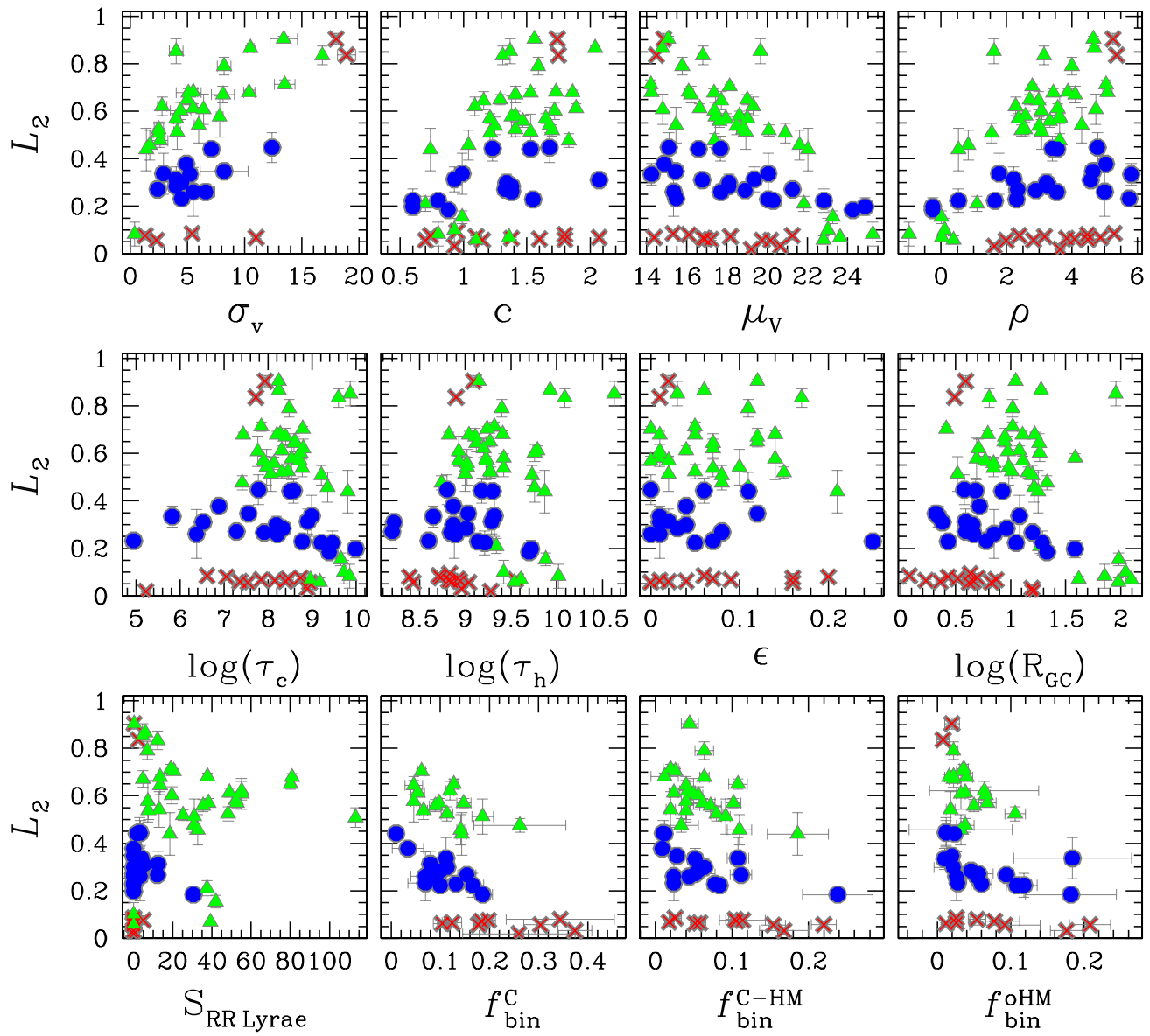

Figure 10. $L 2$ as a function of the same parameters of the host GCs as used in Figure 9.

(A color version of this figure is available in the online journal.)
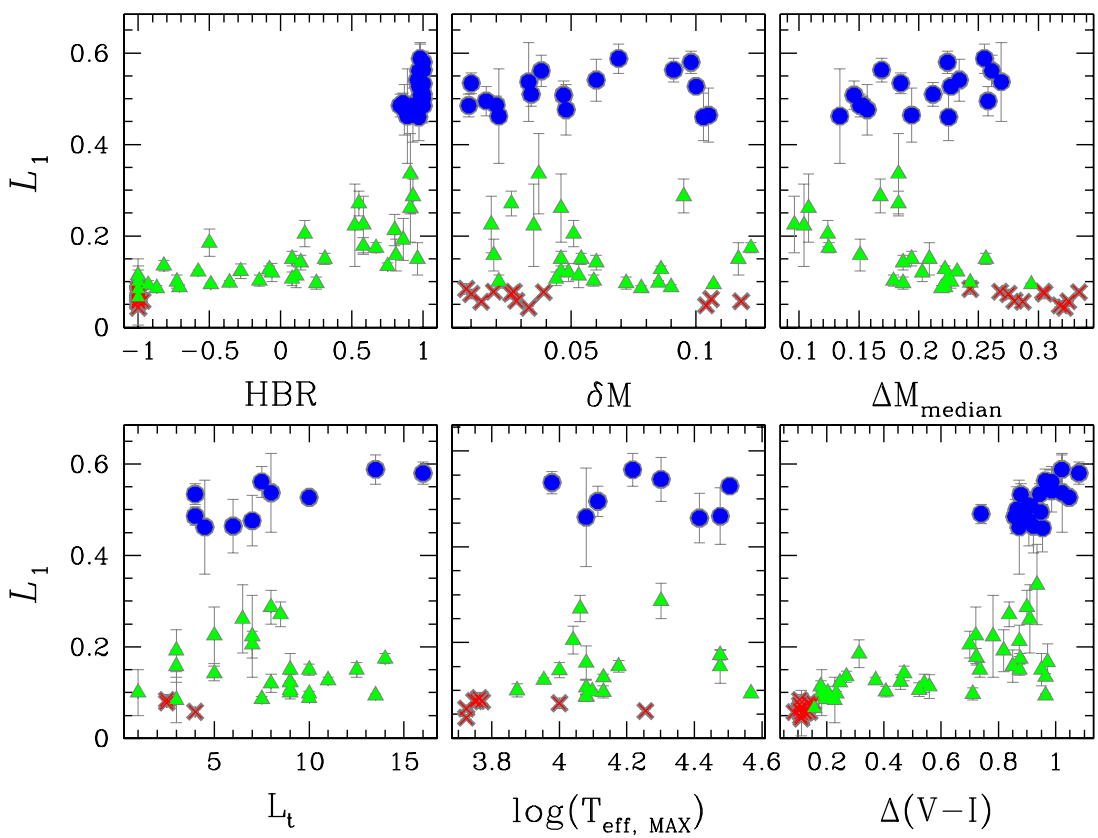

Figure 11. $L 1$ as a function of several quantities used to parameterize HB morphology. Total length of the HB from Fusi Pecci et al. (1993, lower-left panel), logarithm of the maximum temperature along the HB from Recio-Blanco et al. (2006, lower-middle panel), median HB color $(\Delta(V-I))$ from Dotter et al. (2010; lower-right panel), HBR ratio (upper-left panel), and maximum and median mass loss from Gratton et al. (2010; upper-middle and upper-right panel).

(A color version of this figure is available in the online journal.) 

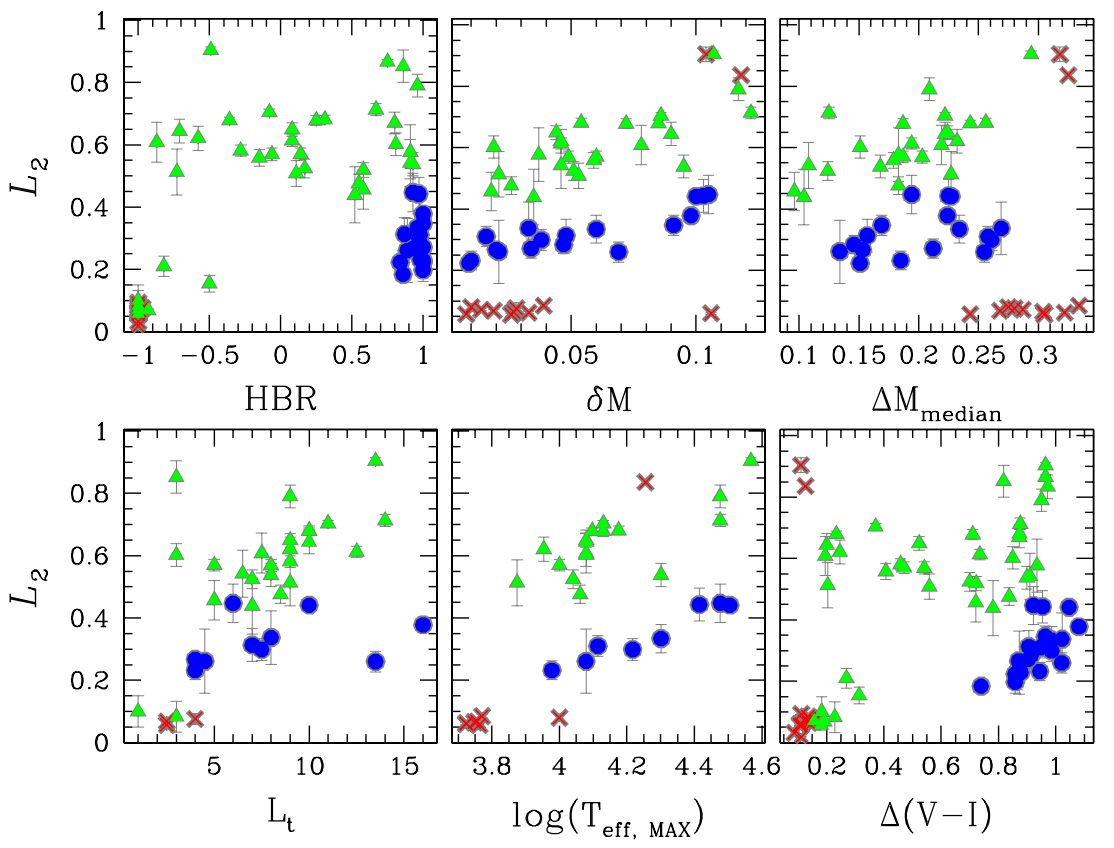

Figure 12. Same as in Figure 11 but for $L 2$.

(A color version of this figure is available in the online journal.)

The wide literature on the 2ndP includes several works, similar to the present investigation, that are based on a fully observational approach, together with others that also use a series of theoretical assumptions. A recent example of the latter is the paper by Gratton et al. (2010), in which the authors used HST/WFPC 2 and ground-based photometry of about 100 GCs to derive median and extreme colors and magnitudes of stars along the HB. They used isochrones and HB evolutionary models to transform these colors into median and extreme masses of stars on the HB and adopted the median mass loss $\left(\Delta M_{\text {median }}=M_{\mathrm{RGB}}-M_{\text {median }}\right)$ and the difference between the median and the minimum HB masses $\left(\delta M=M_{\text {median }}-M_{\min }\right.$, where $M_{\mathrm{RGB}}, M_{\text {median }}$, and $M_{\text {min }}$ are RGB, median, and minimum HB masses, respectively) as parameters of the HB morphology. To determine $\Delta M_{\text {median }}$ and $\delta M$, Gratton et al. (2010) assumed for each cluster the value of metallicity and age from Carretta et al. (2009). They find that the median mass loss correlates with metallicity and suggest that if the mass-loss law they used is universal, age is the 2ndP. They conclude that age can explain the behavior of the median HB when it is coupled with a given mass-loss law that is a linear function of $[\mathrm{Fe} / \mathrm{H}]$. Gratton et al. also suggest that at least another parameter is needed to explain the HB morphology in GCs and argue that He abundance is the most likely candidate. They show that star-to-star helium variations, when combined with a small random quantity, can reproduce the HB morphology, thus supporting the results of other authors (e.g., D'Antona et al. 2002; D'Antona \& Caloi 2008; Dalessandro et al. 2013). They find that the HB extension correlates with the interquartile of the $\mathrm{Na}-\mathrm{O}$ anticorrelation, as previously noticed by the same group of authors (Carretta et al. 2007). Figures 11 and 12 show that $\delta M$ correlates with $L 2$ in G2 and G3 GCs, while there is no significant correlation between $L 2$ and $\Delta M_{\text {median }}$. The relation between $L 1$ and $\Delta M_{\text {median }}$ is similar to that of $L 1$ and $[\mathrm{Fe} / \mathrm{H}]$. This reflects the tight correlation between $\Delta M_{\text {median }}$ and metallicity.

Fusi Pecci et al. (1993) analyzed 53 GCs and found that the net length $\left(L_{\mathrm{t}}\right)$ of the HB and the presence and extent of blue tails are correlated with the GC density and concentrations,

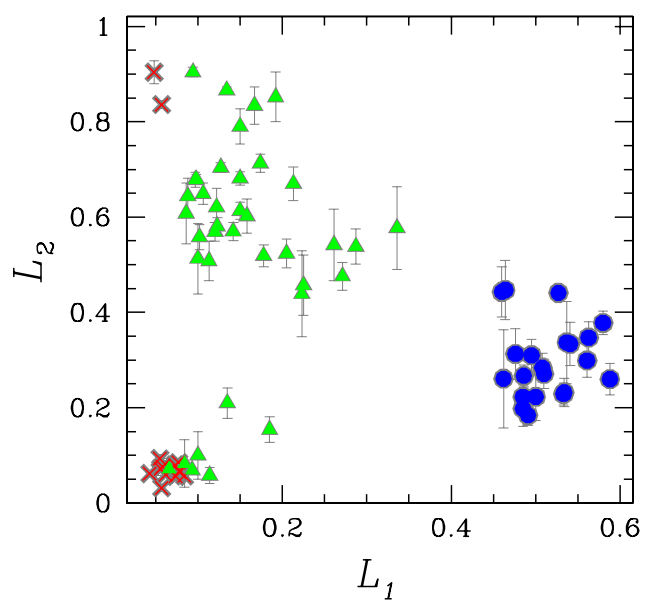

Figure 13. $L 2$ as a function of $L 1$ for the $74 \mathrm{GCs}$ studied in this paper.

(A color version of this figure is available in the online journal.)

with more concentrated or denser GCs having bluer and longer HB morphologies. A correlation between HB morphology and absolute magnitude has been also detected by Recio-Blanco et al. (2006), who analyzed the CMDs of 54 GCs obtained from homogeneous HST WFPC2 data (Piotto et al. 2002) and concluded that the maximum effective temperature ( $T_{\text {eff, }}$ MAX $)$ encountered along the $\mathrm{HB}$ correlates with $M_{\mathrm{V}}$, with moreluminous GCs having also more-extended $\mathrm{HBs}$.

As discussed in Section 1, the way an HB morphology metric is defined influences the outcome. It explains why some studies conclude that mass and/or He content are the main driver of $\mathrm{HB}$ morphology, while others indicate age as the main 2 ndP. The definition of the two parameters $L 1$ and $L 2$ and their comparison with other quantities commonly used to parameterize the $\mathrm{HB}$ morphology (like $L_{\mathrm{t}}, T_{\mathrm{eff}, \mathrm{MAX}}$, and $\Delta(V-I)$ ) may help to shed some light on this controversy. Figure 11 shows that on the one hand there is no significant correlation between either $L_{\mathrm{t}}$ or $T_{\text {eff,MAX }}$ and $L 1$. On the other hand, among G2 GCs, $L 1$ 
correlates with $\Delta(V-I)$, and G3 GCs have, on average, larger $L 1$ values than G2 GCs (indicative of older ages in G3 than G2). From Figure 12 we note that $L 2$ correlates with $T_{\text {eff,MAX }}$ for both G2 and G3 GCs, and a correlation between $L 2$ and $L_{\mathrm{t}}$ is also observed for G2 GCs. Figure 12 reveals no significant correlation between $L 2$ and $\Delta(V-I)$. Finally, in Figure 13 we compare the two parameters introduced in this paper and show that $L 2$ and $L 1$ are not significantly correlated.

We conclude that both the metric defined by Fusi Pecci et al. (1993) and Recio-Blanco et al. (2006), as well as L2, are sensitive to some properties (possibly helium variations) of the HB morphology but lack sensitivity to others (such us age). In contrast, $\Delta(V-I)$ and $L 1$ are more sensitive to different properties of the HB (e.g., metallicity and age). The use of a pair of parameters, such as $L 1$ and $L 2$, can provide a more exhaustive description of the HB morphology than one alone.

\section{SUMMARY AND CONCLUSIONS}

In this paper we exploit both recent observational findings and ideas provided in the early 1980s to investigate the relation between HB morphology and various properties in GCs. These new findings come from studies on multiple stellar populations in GCs that show that the position of a star along the $\mathrm{HB}$ is connected to its chemical composition. First-generation stars populate the cooler side of the HB, and second-generation (He-enriched) stars populate the hotter side.

Freeman \& Norris (1981) suggested that apart from metallicity, at least two parameters are needed to explain the HB morphology. One of these should be a global parameter that varies from GC to GC and the other a nonglobal parameter that varies within the GC. Driven by this idea we defined two new parameters to describe the HB morphology: $L 1$, which indicates the distance between the RGB and the coolest part or the HB, and $L 2$, which measures the color extension of the HB. Our analysis reveals that $L 1$ depends on GC age and metallicity, while $L 2$ correlates with the GC luminosity (hence, the mass) and the range of He content $(\Delta Y)$.

These results suggest that, along the lines suggested by Freeman \& Norris, age and metallicity are the main global parameters of the HB morphology of GCs, while GC mass is related to the $\mathrm{HB}$ extension. Works on multiple stellar populations in GCs show that more massive GCs exhibit, on average, larger internal helium variations, $\Delta Y$, than less massive GCs; $\Delta Y$ is positively correlated with $L 2$ and GC mass, though this analysis is limited to a small number of GCs at present. This makes it very tempting to suggest that internal star-to-star helium variation, associated with GC mass and the presence of multiple populations, is the main nonglobal parameter. ${ }^{15}$ The use of two quantities $L 1$ and $L 2$, that share a common definition (Section 3) but are sensitive to different phenomena allow us to discriminate the effects of global and nonglobal parameters on the HB morphology.

We thank F. D'Antona and J. Lattanzio for useful discussion and suggestion. We are grateful to P. Stetson for providing unpublished light curves of RR Lyrae. A.P.M., J.E.N., and H.J. acknowledge the financial support from the Australian Research Council through Discovery Project grant DP120100475.

\footnotetext{
15 If this scenario is correct, in some metal-intermediate GCs with very small L 2 values, like AM-1, Eridanus, Pal 3, Pal 4, Pal 14, Rup 106, and Pyxis, any internal helium variation, if present, should be very small. These GCs are hence good candidates to host a simple stellar population.
}

J.E.N. is also supported by the Australian Research Council through Discovery Project grant DP0984924. A.D. and M.A. acknowledge support from the Australian Research Council (grant FL110100012). G.P. and S.C. acknowledge financial support from PRIN MIUR 2010-2011, project "The Chemical and Dynamical Evolution of the Milky Way and Local Group Galaxies," prot. 2010LY5N2T, and from PRIN-INAF 2011 "Multiple Populations in Globular Clusters: their role in the Galaxy assembly." Support for this work has been provided by the IAC (grant 310394) and the Education and Science Ministry of Spain (grants AYA2007-3E3506, and AYA2010-16717).

\section{APPENDIX}

\section{THE IMPACT OF THE RR LYRAE PHASE ON $L 1$ AND L2 MEASUREMENTS}

To investigate the impact of excluding RR Lyrae that might be out of the instability strip in the determination of $L 1$ and $L 2$, we have simulated a number of CMDs for different choices of the fraction of RR Lyrae $\left(f_{\mathrm{V}}\right)$, red-HB $\left(f_{\mathrm{R}}\right)$, and blue-HB $\left(f_{\mathrm{B}}\right)$ stars. We assumed that RR Lyrae are distributed along the whole instability strip and that all the RR Lyrae ab have the same light curve (this corresponds to the light curve observed for V27 in M 4, $P \sim 0.612$ day, and is one of the RR Lyrae ab in M 4). We assumed for all the RR Lyrae $\mathrm{c}$ the light curve of V40 in M 4, $P \sim 0.299$ day. The reason why we have chosen these two RR Lyrae is that their amplitude and period are typical of RR Lyrae $a b$ and c. The light curves have been kindly provided by Peter Stetson. They are based on more than 1000 observations in $B, V$, and $R$ bands and have been converted into F606W and F814W by using the color-temperature relations by Dotter et al. (2008). We choose V40, for which we have a light curve with the largest amplitude available to us, to maximize possible effects on the determination of $L 1$ and $L 2$.

F606W and F814W magnitudes have been simulated at different phases to account for the fact that F606W and F814W images are taken at different times. We assumed $f_{\mathrm{R}}=N \times$ $\left(f_{\mathrm{B}}+f_{\mathrm{R}}\right)(N=0.0,0.04,0.10,0.50,0.90,0.96,1.0)$ and $f_{\mathrm{V}}=$ $M \times\left(f_{\mathrm{B}}+f_{\mathrm{R}}+f_{\mathrm{V}}\right)(M=0,0.10,0.25,0.50)$ and simulated 1000 CMDs for each combination of $f_{\mathrm{B}}, f_{\mathrm{V}}, f_{\mathrm{R}}$. For each CMD we have calculated $L 1_{\mathrm{I}}, L 2_{\mathrm{I}}$ and $L 1_{\mathrm{II}}, L 2_{\mathrm{II}}$. These are the values of $L 1$ and $L 2$ obtained when all RR Lyrae lie within the instability strip and when RR Lyrae are at random phase, respectively. The differences $\Delta L 1=L 1_{\mathrm{I}}-L 1_{\mathrm{II}}$ and $\Delta L 2=L 2_{\mathrm{I}}-L 2_{\mathrm{II}}$ are maximal in the case of an HB made of RR Lyrae only $(\Delta L 1=$ $0.10, \Delta L 2=0.22)$. Large difference are also detected in the case of an HB with a very small fraction of red HB stars and a large fraction of RR Lyrae variables $\left(f_{\mathrm{R}}=0\right.$ or $\left.f_{\mathrm{R}}=0.04\right)$. We obtain $\Delta L 1=\Delta L 2 \sim 0.08$ when assuming $f_{\mathrm{V}}=0.50$; $\Delta L 1=\Delta L 2 \sim 0.04$ for $f_{\mathrm{V}}=0.25$; and $\Delta L 1=\Delta L 2 \sim 0.01$ for $f_{\mathrm{V}}=0.1$. We obtain similar results for $\Delta L 2$ and $\Delta L 1 \sim 0$ when the blue HB hosts a very small fraction of stars $\left(f_{\mathrm{B}}=0\right.$ and $f_{\mathrm{B}}=$ $0.04)$. The $\Delta L 1$ and $\Delta L 2$ get closer to zero for larger values of $f_{\mathrm{B}}$ and $f_{\mathrm{R}}$.

According to the literature values (Lee et al. 1994; Harris 1996, 2010 edition; Gratton et al. 2010, and references therein), extreme cases of $f_{\mathrm{V}} \geqslant 0.25$ and $f_{\mathrm{R}} \leqslant 0.1$ are not present among the clusters studied in this paper, which suggests that any error related to the RR Lyrae phase should be smaller than 0.03-0.04 mag.

As a further test, for each GC studied in this paper we assumed the corresponding values of $f_{\mathrm{B}}, f_{\mathrm{V}}$, and $f_{\mathrm{R}}$ (Lee et al. 1994; Harris 1996, 2003 edition, and references therein) and 
simulated 10,000 CMDs. For most GCs we found $\Delta L 1<0.02$ and $\Delta L 2<0.02 ; \Delta L 1$ is greater than 0.03 mag only in a few GCs, namely NGC 4590, NGC 7078, and NGC 5466. The $\Delta L 2$ exceeds 0.03 mag also in the cases of Pal 3 and Rup 106. Both $\Delta L 1$ and $\Delta L 2$ never exceed $0.04 \mathrm{mag}$. Our tests suggest that the uncertainties on $L 1$ and $L 2$ measurements due to the random phase of RR Lyrae should be negligible for our purposes. This conclusion is similar to that of Gratton et al. (2010), who showed that RR Lyrae should not affect the determination of the median colors of HB stars.

\section{REFERENCES}

Alves-Brito, A., Yong, D., Meléndez, J., Vásquez, S., \& Karakas, A. I. 2012, A\&A, 540, A3

Anderson, J., Sarajedini, A., Bedin, L. R., et al. 2008, AJ, 135, 2055

Arp, H. C., Baum, W. A., \& Sandage, A. R. 1952, AJ, 57, 4

Bellini, A., Bedin, L. R., Piotto, G., et al. 2010, AJ, 140, 631

Bellini, A., Piotto, G., Milone, A. P., et al. 2013, ApJ, 765, 32

Caloi, V., \& D'Antona, F. 2008, ApJ, 673, 847

Carretta, E. 2006, AJ, 131, 1766

Carretta, E., Bragaglia, A., Gratton, R. G., et al. 2010a, A\&A, 520, A95

Carretta, E., Bragaglia, A., Gratton, R. G., et al. 2010b, A\&A, 516, A55

Carretta, E., Bragaglia, A., Gratton, R., D’Orazi, V., \& Lucatello, S. 2009, A\&A, 508,695

Carretta, E., Recio-Blanco, A., Gratton, R. G., Piotto, G., \& Bragaglia, A. 2007, ApJL, 671, L125

Cassisi, S., Salaris, M., Pietrinferni, A., et al. 2008, ApJL, 672, L115

Cassisi, S., Salaris, M., \& Pietrinferni, A. 2012, MmSAI, 84, 91

Catelan, M. 2000, ApJ, 531, 826

Catelan, M. 2009, Ap\&SS, 320, 261

Catelan, M., \& de Freitas Pacheco, J. A. 1993, AJ, 106, 1858

Dalessandro, E., Salaris, M., Ferraro, F. R., Mucciarelli, A., \& Cassisi, S. 2013, MNRAS, 430, 459

D’Antona, F., Bellazzini, M., Caloi, V., et al. 2005, ApJ, 631, 868

D'Antona, F., \& Caloi, V. 2008, MNRAS, 390, 693

D’Antona, F., Caloi, V., Montalbán, J., Ventura, P., \& Gratton, R. 2002, A\&A, 395,69

De Angeli, F., Piotto, G., Cassisi, S., et al. 2005, AJ, 130, 116

di Criscienzo, M., D’Antona, F., Milone, A. P., et al. 2011, MNRAS, 414, 3381

di Criscienzo, M., Ventura, P., D'Antona, F., Milone, A., \& Piotto, G. 2010, MNRAS, 408, 999

Djorgovski, S., \& Meylan, G. 1994, AJ, 108, 1292

Dotter, A., Chaboyer, B., Jevremović, D., et al. 2008, ApJS, 178, 89

Dotter, A., Sarajedini, A., Anderson, J., et al. 2010, ApJ, 708, 698

Dotter, A., Sarajedini, A., \& Anderson, J. 2011, ApJ, 738, 74

Ferraro, F. R., Paltrinieri, B., Pecci, F. F., Rood, R. T., \& Dorman, B. 1998, ApJ, 500,311

Freeman, K. C., \& Norris, J. 1981, ARA\&A, 19, 319

Fusi Pecci, F., Ferraro, F. R., Bellazzini, M., et al. 1993, AJ, 105, 1145

Fusi-Pecci, F., \& Renzini, A. 1978, in IAU Symp. 80, The HR Diagram-the 100th Anniversary of Henry Norris Russell, ed. A. G. D. Philip \& D. S. Hayes (Dordrecht: Reidel), 225

Gratton, R. G., Carretta, E., Bragaglia, A., Lucatello, S., \& D'Orazi, V. 2010, A\&A, 517, A81

Gratton, R. G., Lucatello, S., Carretta, E., et al. 2011, A\&A, 534, A123

Gratton, R. G., Lucatello, S., Carretta, E., et al. 2012, A\&A, 539, A19

Gratton, R. G., Lucatello, S., Sollima, A., et al. 2013, A\&A, 549, A41

Harris, W. E. 1996, AJ, 112, 1487

Iben, I. 1968, Natur, 220, 143
King, I. 1962, AJ, 67, 471

King, I. R., Bedin, L. R., Cassisi, S., et al. 2012, AJ, 144, 5

Kraft, R. P. 1994, PASP, 106, 553

Leaman, R., VandenBerg, D. A., \& Mendel, J. T. 2013, MNRAS, 436, 122

Lee, Y.-W., Demarque, P., \& Zinn, R. 1994, ApJ, 423, 248

Lind, K., Charbonnel, C., Decressin, T., et al. 2011, A\&A, 527, A148

Lovisi, L., Mucciarelli, A., Lanzoni, B., et al. 2012, ApJ, 754, 91

Marín-Franch, A., Aparicio, A., Piotto, G., et al. 2009, ApJ, 694, 1498

Marino, A. F., Milone, A. P., \& Lind, K. 2013, ApJ, 768, 27

Marino, A. F., Milone, A. P., Piotto, G., et al. 2009, A\&A, 505, 1099

Marino, A. F., Milone, A. P., Przybilla, N., et al. 2014, MNRAS, 437, 1609

Marino, A. F., Milone, A. P., Sneden, C., et al. 2012, A\&A, 541, A15

Marino, A. F., Sneden, C., Kraft, R. P., et al. 2011a, A\&A, 532, A8

Marino, A. F., Villanova, S., Milone, A. P., et al. 2011b, ApJL, 730, L16

Marino, A. F., Villanova, S., Piotto, G., et al. 2008, A\&A, 490, 625

Mengel, J. G., \& Gross, P. G. 1976, Ap\&SS, 41, 407

Milone, A. P., Bedin, L. R., Piotto, G., et al. 2008, ApJ, 673, 241

Milone, A. P., Marino, A. F., Piotto, G., et al. 2012a, ApJ, 745, 27

Milone, A. P., Marino, A. F., Piotto, G., et al. 2013, ApJ, 767, 120

Milone, A. P., Piotto, G., Bedin, L. R., et al. 2012b, A\&A, 540, A16

Milone, A. P., Piotto, G., Bedin, L. R., et al. 2012c, A\&A, 537, A77

Milone, A. P., Piotto, G., Bedin, L. R., et al. 2012d, ApJ, 744, 58

Monelli, M., Milone, A. P., Stetson, P. B., et al. 2013, MNRAS, 431, 2126

Norris, J. 1981, ApJ, 248, 177

Norris, J. 1983, ApJ, 272, 245

Norris, J. 1987, ApJL, 313, L65

Norris, J., Cottrell, P. L., Freeman, K. C., \& Da Costa, G. S. 1981, ApJ, 244, 205

Peterson, R. C. 1982, ApJ, 258, 499

Piotto, G., Bedin, L. R., Anderson, J., et al. 2007, ApJL, 661, L53

Piotto, G., King, I. R., Djorgovski, S. G., et al. 2002, A\&A, 391, 945

Piotto, G., Milone, A. P., Anderson, J., et al. 2012, ApJ, 760, 39

Piotto, G., Milone, A. P., Marino, A. F., et al. 2013, ApJ, 775, 15

Recio-Blanco, A., Aparicio, A., Piotto, G., de Angeli, F., \& Djorgovski, S. G. 2006, A\&A, 452, 875

Rood, R. T., \& Seitzer, P. O. 1981, in IAU Colloq. 68: Astrophysical Parameters for Globular Clusters, ed. A. G. D. Philip \& D. S. Hayes (Schenectady: Davis Press), 369

Rosenberg, A., Saviane, I., Piotto, G., \& Aparicio, A. 1999, AJ, 118, 2306

Salaris, M., Riello, M., Cassisi, S., \& Piotto, G. 2004, A\&A, 420, 911

Sandage, A., \& Wallerstein, G. 1960, ApJ, 131, 598

Sandage, A., \& Wildey, R. 1967, ApJ, 150, 469

Sarajedini, A., Bedin, L. R., Chaboyer, B., et al. 2007, AJ, 133, 1658

Searle, L., \& Zinn, R. 1978, ApJ, 225, 357

Shetrone, M. D., \& Keane, M. J. 2000, AJ, 119, 840

Siess, L., \& Livio, M. 1999, MNRAS, 308, 1133

Sirianni, M., Jee, M. J., Benítez, N., et al. 2005, PASP, 117, 1049

Smith, G. H., \& Mateo, M. 1990, ApJ, 353, 533

Smith, G. H., \& Norris, J. E. 1993, AJ, 105, 173

Sneden, C., Kraft, R. P., Guhathakurta, P., Peterson, R. C., \& Fulbright, J. P. 2004, AJ, 127, 2162

Soker, N. 1998, AJ, 116, 1308

Stetson, P. B., Bolte, M., Harris, W. E., et al. 1999, AJ, 117, 247

van den Bergh, S. 1965, JRASC, 59, 151

van den Bergh, S. 1967, AJ, 72, 324

Vandenberg, D. A., Brogaard, K., Leaman, R., \& Casagrande, L. 2013, ApJ, 775,134

Ventura, P., Caloi, V., D’Antona, F., et al. 2009, MNRAS, 399, 934

Ventura, P., \& D’Antona, F. 2005, ApJL, 635, L149

Villanova, S., Geisler, D., \& Piotto, G. 2010, ApJL, 722, L18

Villanova, S., Piotto, G., \& Gratton, R. G. 2009, A\&A, 499, 755

Yong, D., Grundahl, F., D’Antona, F., et al. 2009, ApJL, 695, L62

Yong, D., Grundahl, F., Johnson, J. A., \& Asplund, M. 2008, ApJ, 684, 1159 